\title{
ADAPTIVE STABILIZATION OF CONTINUOUS-TIME SYSTEMS THROUGH A CONTROLLABLE MODIFIED ESTIMATION MODEL
}

\author{
M. DE LA SEN
}

Received 9 October 2003 and in revised form 9 February 2004

This paper presents an indirect adaptive control scheme of continuous-time systems. The estimated plant model is controllable and then the adaptive scheme is free from singularities. Such singularities are avoided through a modification of the estimated plant parameter vector so that its associated Sylvester matrix is guaranteed to be nonsingular. That property is achieved by ensuring that the absolute value of its determinant does not lie below a positive threshold. An alternative modification scheme based on the achievement of a modified diagonally dominant Sylvester matrix of the parameter estimates is also proposed. This diagonal dominance is achieved through estimates modification as a way to guarantee the controllability of the modified estimated model when a controllability measure of the estimation model without modification fails. In both schemes, the use of an explicit hysteresis switching function for the modification of the estimates is not required to ensure the controllability of the modified estimated model. Both schemes ensure that chattering due to switches associated with the modification is not present.

\section{Introduction}

The adaptive stabilization and control of linear continuous and discrete systems have been successfully developed in the last decades [10, 17, 18, 19]. Usually, the plant is assumed to be inversely stable and its relative degree and its high-frequency gain sign are assumed to be known together with an absolute upper-bound for that gain in the discrete case. The assumption on the knowledge of the order can be relaxed by assuming a nominal known order and considering the exceeding modes as unmodeled dynamics $[2,4,5,11,16]$. The assumption on the knowledge of the high-frequency gain has been removed in $[13,19]$ and the assumption of the plant being inversely stable has been successfully removed in the discrete case and, more recently, in the continuous one $[2,5,6,8,11]$. The problem is solved by using either excitation of the plant signals or a modification of the least-squares estimation by either using excitation of the plant signals or exploiting the properties of the standard least-squares covariance matrix $[2,3,4,5,6,11]$. In a set of 
papers, the assumption of the plant being inversely stable has been removed by using either excitation of the plant signals or estimates modification by using hysteresis switching functions which generate the controllability of the estimated plant model while exploiting the properties of the covariance matrix $([4,5,6]$ and the references therein focused on a deterministic approach). An alternative modification strategy was the use of a random search-type algorithm to avoid the degeneracy of the Sylvester matrix [11]. In [2], a recursive coordinate modification method was given, which ensues convergence in a stochastic sense. This paper presents a pole/placement-based adaptive stabilization algorithm for continuous-time systems which can have unstable zeros. The adaptive scheme uses a parameter modification scheme which neither involves an explicit hysteresis switching rule nor takes advantage of the properties of the covariance matrix, while guarantees that the absolute value of the determinant of the Sylvester matrix associated with the parameter estimates is bounded from below by a positive threshold. An alternative modification procedure which is based upon the achievement of a diagonally dominant Sylvester matrix of the modified estimates is also proposed. The mechanism which guarantees the controllability of the modified estimated plant model consists basically in the online perturbation of some of the estimated plant parameters prior to the modification. In this way, the resulting modified Sylvester matrix becomes nonsingular, while chattering is avoided since the control law based on the modified estimates is nonsingular and then solvable for all time $[14,15]$ when the controllability of the estimation model fails against some appropriate numerical test about nonsingularity. The estimation scheme has suitable stability and convergence properties, and the resulting closed-loop scheme is asymptotically stable in the large in the absence of noise and unmodeled dynamics. This modification is an alternative method in the case when a sufficiency test on maintenance of controllability of the unmodified estimated model fails. Such a test consists in guaranteeing through the manipulation of matrix norms that the maximum absolute eigenvalue of the Sylvester matrix of such a model is bounded from above by a finite real constant while the minimum one is bounded from below by a positive real constant. The boundedness and convergence of all the estimates and controller parameters are guaranteed in both the ideal perfectly modeled case and when the wide class of unmodeled dynamics and bounded disturbances considered in $[4,5,16]$ is present. The plant input and output are bounded and converge to zero in the ideal perfectly modeled case, while they are bounded in the above-mentioned nonideal situation. Section 2 is devoted to the synthesis of the adaptive stabilizer in the perfectly modeled case for unknown continuous-time plants. The basic estimation scheme, used prior to the modification procedure, is of least-squares type. The above-mentioned two estimation modification procedures are also given. Section 3 presents the convergence and stability properties of the proposed scheme. Some robustness issues against the presence of unmodeled dynamics and bounded disturbances are also pointed out. The mechanism used to guarantee robustness is the variation of the basic estimation scheme by adding a relative dead zone so that the estimation and covariance matrix adaptation are frozen when the adaptation error is small compared to an absolute overbounding function of the contribution to the uncertainties to the output. The modification procedures that ensure controllability of the estimated model are kept as in the ideal case. The scheme's modifications to operate in the case of the presence of 
unmodeled dynamics and/or bounded disturbances are also given. A numerical example is given in Section 4 and, finally, conclusions end the paper. The mathematical proofs of the results are developed in the appendix.

\section{Adaptive stabilizer for a continuous-time plant}

In the sequel, the time-argument is suppressed unless confusion can arise and the constant parameters are denoted by a superscript “ $*$ ". Consider the following continuoustime controllable system:

$$
A^{*}(D) y(t)=B^{*}(D) u(t), \quad D^{i} y(0)=y_{0}^{(i)} \quad(i=0,1, \ldots, n-1)
$$

where $D^{i} \equiv d^{i} / d t^{i}(i=0,1, \ldots, n-1)$ is the $i$ th time-derivative operator and $A^{*}(D)=D^{n}+$ $\sum_{i=1}^{n} a_{i}^{*} D^{n-i}$ and $B^{*}(D)=\sum_{i=0}^{m} b_{i}^{*} D^{m-i}$ with $n \geq m$. Since (2.1) is controllable, then its associated $(n+m)$ Sylvester resultant matrix

$$
S\left(\theta_{0}^{*}\right)=\left[\begin{array}{ccccccc}
1 & 0 & \cdots & 0 & b_{0}^{*} & \cdots & 0 \\
a_{1}^{*} & 1 & & \vdots & b_{1}^{*} & & 0 \\
\vdots & a_{1}^{*} & & 0 & b_{m}^{*} & & \vdots \\
a_{n}^{*} & \vdots & \ddots & 1 & \vdots & \ddots & b_{0}^{*} \\
0 & a_{n}^{*} & & a_{1}^{*} & & & b_{1}^{*} \\
\vdots & 0 & \ddots & \vdots & 0 & \ddots & \vdots \\
0 & \cdots & \cdots & a_{n}^{*} & 0 & \cdots & b_{m}^{*}
\end{array}\right]
$$

is nonsingular. Define the filtered signals

$$
E^{*}(D) u_{f}=u, \quad E^{*}(D) y_{f}=y, \quad E^{*}(D)=D^{n}+\sum_{i=1}^{n-1} e_{i}^{*} D^{n-i}
$$

with $E^{*}(D)$ being a strictly Hurwitz polynomial of (maximum) degree $n$. For simplicity in the mathematical developments, it is assumed in the following that such a degree is $n$ with no loss in generality. The filtered control law for a known plant (2.1) is generated as

$$
S^{*}(D) u_{f}=-R^{*}(D) y_{f},
$$

where $S^{*}(D) u_{f}=-R^{*}(D) y_{f}, R^{*}(D)=D^{m}+\sum_{i=0}^{m-1} r_{i}^{*} D^{m-i-1}$ satisfy the Diophantine equation $A^{*}(D) S^{*}(D)+B^{*}(D) R^{*}(D)=C^{*}(D)$, where $C^{*}(D)=D^{n}+\sum_{i=1}^{n^{*}-1} c_{i}^{*} D^{n^{*}-i}$ of prefixed degree fulfilling the constraint $n^{*} \leq n+\operatorname{deg}\left(S^{*}(D)\right)=2 n$ is a strictly Hurwitz polynomial (i.e., with roots in $\operatorname{Re} D<0$ ) which defines the suited closed-loop dynamics. The polynomials $S^{*}(D)$ and $R^{*}(D)$ are the unique solution to the above Diophantine equations since $A^{*}(D)$ and $B^{*}(D)$ are coprime because of the controllability of $(2.1)$ and the constraints $\operatorname{deg}\left(S^{*}(D)\right) \leq \operatorname{deg}\left(E^{*}(D)\right)<n$ and $\operatorname{deg}\left(R^{*}(D)\right)<\operatorname{deg}\left(A^{*}(D)\right)$. (In particular, if $E^{*}(D)$ would satisfy $\operatorname{deg}\left(E^{*}(D)\right) \leq n-1$, then its appropriate coefficients in $(2.3)$ 
will be zeroed in (2.3).) Equation (2.4) is equivalent to its unfiltered version

$$
u=\left(E^{*}(D)-S^{*}(D)\right) u_{f}-R^{*}(D) y_{f} .
$$

The control objective in the adaptive case for unknown plant is to update the controller parameters $s_{i}$ and $r_{j}(i=1,2, \ldots, n, j=0,1, \ldots, m)$ in an adaptive way so that the plant (2.1), subject to the control law (2.5) when replacing the parameters by their estimates, is asymptotically stable in the large in the absence of disturbances. Under bounded noise and a standard class of unmodeled dynamics, the scheme is guaranteed to be globally stable. Simple direct calculus with (2.1) and (2.3) yields, for filtered signals,

$$
D^{n} y_{f}=\theta^{* T} \varphi
$$

with

$$
\begin{aligned}
\theta^{*} & =\left[\theta_{0}^{* T}, \varepsilon_{0}^{* T}\right]^{T}=\left[\theta_{1}^{*}, \theta_{2}^{*}, \ldots, \theta_{n+m+1}^{*}, \theta_{n+m+2}^{*}, \theta_{n+m+3}^{*}, \ldots, \theta_{2 n+m+1}^{*}\right]^{T} \\
& =\left[b_{0}^{*}, b_{1}^{*}, \ldots, b_{m}^{*}, a_{1}^{*}, a_{2}^{*}, \ldots, a_{n}^{*}, \varepsilon_{01}^{*}, \varepsilon_{02}^{*}, \ldots, \varepsilon_{0 n}^{*}\right]^{T} \\
\varphi(t) & =\left[\varphi_{0}^{T}(t), i_{\varphi}^{T}(t)\right]^{T}, \\
& =\left[D^{m} u_{f}, D^{m-1} u_{f}, \ldots, u_{f},-D^{n-1} y_{f},-D^{n-2} y_{f}, \ldots,-y_{f}, i_{1}, i_{2}, \ldots, i_{n}\right]^{T},
\end{aligned}
$$

where $g(t)=\varepsilon_{0}^{T}(t) i(t)$ is an exponentially decaying term that depends on initial conditions, and each $i_{j}(t)$ is known and it has the form $t^{\ell} e^{\lambda_{k}^{*} t}$ for $\ell=0,1, \ldots, m_{k}-1$ with $m_{k}$ being the multiplicity of the root $\lambda_{k}^{*}$ of $C^{*}(D)$. There are $m_{k}$ terms $i_{(\cdot)}(t)$ of such a form for each $\lambda_{k}^{*}$. The parameter vector $\theta^{*}$ is estimated by using standard least-squares algorithms of covariance matrix $P(t)$ and estimated vector $\theta(t)=\left(\theta_{0}^{T}(t), \varepsilon_{0}^{T}(t)\right)^{T}$ with $\varepsilon_{0}(t)$ being the estimation of the initial conditions of $\varepsilon_{0}^{*}$. The estimation algorithm consists of an estimation algorithm and a rule to modify such estimates as follows.

\subsection{Parameter estimation.}

$$
\begin{aligned}
& e=D^{n} y_{f}-\theta^{T} \varphi \quad \text { (prediction error), } \\
& \dot{\theta}=P \varphi e, \\
& \dot{P}=-P \varphi \varphi^{T} P, \quad P(0)=P^{T}(0)>0 .
\end{aligned}
$$

The basic modification of the estimated plant model is performed when necessary to maintain the controllability of the estimated model in the sense that $\left|\operatorname{Det}\left(S\left(\bar{\theta}_{0}\right)\right)\right| \geq \rho>0$ even if $\left|\operatorname{Det}\left(S\left(\theta_{0}\right)\right)\right|<\rho$ for some positive real constant $\rho$, while the Sylvester matrices of the a priori and modified estimates have the same structures as $S\left(\theta_{0}^{*}\right)$ and their values are obtained by replacing $\theta_{0}^{*}$ with $\theta_{0}$ and $\bar{\theta}_{0}$, respectively. The idea behind the modification method, formulated below in (2.9) and (2.10), is basically as follows. Two different thresholds are used to modify the parameter components. The use of two thresholds $\rho$ and $\rho^{\prime}$ is only made for purposes of avoiding chattering by involving the mechanism of switching between them each time a discontinuity in the modification is found. These thresholds are sufficiently small compared to the stability abscissa of the objective polynomial $C^{*}(D)$ in order to guarantee the closed-loop stability. Each absolute value of 
a parameter estimate is either modified with a maximum amount $\alpha(t)$ or such a parameter becomes unmodified (see (2.10)). The maximum value of $\alpha(t)$ depends on the thresholds

$\rho$ and $\rho^{\prime}$ (see (2.9a), (2.9b), and (2.9c)). The mechanism which ensures that the absolute value modified Sylvester determinant exceeds the corresponding threshold is to manipulate its Taylor expansion around its unmodified value by checking the maximum allowable absolute increase by increasing each of all the estimates in $\pm \alpha$ or leaving them unmodified, see (2.9d), (2.9e), (2.9f), and (2.9g). The modification scheme for calculating $\bar{\theta}$ from $\theta$ is implemented according to the following scheme.

2.2. Basic modification of the estimation. The plant parameter estimates through the algorithm (2.8) are then modified as follows. First, define the strictly positive piecewise constant real time-function $h(\cdot)$ and nonnegative time-functions $\delta_{\alpha}$ and $\alpha$ as follows, for positive real constants $\rho$ and $\rho^{\prime} \geq \rho$ :

$$
\begin{gathered}
h(0)=\rho, \quad h\left(t^{+}\right)= \begin{cases}\rho & \text { if } h(t)=\rho,\left|\operatorname{Det}\left(S\left(\theta_{0}\right)\right)\right| \neq \rho \text { for } t=t^{-}, \\
\rho & \text { if } h(t)=\rho^{\prime},\left|\operatorname{Det}\left(S\left(\theta_{0}\right)\right)\right|=\rho^{\prime} \text { for } t=t^{-}, \\
\rho^{\prime} & \text { if } h(t)=\rho^{\prime},\left|\operatorname{Det}\left(S\left(\theta_{0}\right)\right)\right| \neq \rho^{\prime} \text { for } t=t^{-}, \\
\rho^{\prime} & \text { if } h(t)=\rho,\left|\operatorname{Det}\left(S\left(\theta_{0}\right)\right)\right|=\rho \text { for } t=t^{-},\end{cases} \\
\delta_{\alpha}= \begin{cases}\frac{3 h-\operatorname{Det}\left(S\left(\theta_{0}\right)\right)}{\bar{C}}=\frac{3 h-\left|\operatorname{Det}\left(S\left(\theta_{0}\right)\right)\right| \operatorname{Sign}(\bar{C}) \operatorname{Sign}\left(\operatorname{Det}\left(S\left(\theta_{0}\right)\right)\right)}{|\bar{C}|} & \text { if }\left|\operatorname{Det} S\left(\theta_{0}\right)\right|<h, \\
0 \quad \text { if }\left|\operatorname{Det} S\left(\theta_{0}\right)\right| \geq h,\end{cases} \\
\alpha= \begin{cases}\delta_{\alpha} \bar{C} & \text { if } \delta_{\alpha} \bar{C} \geq 1, \\
\left(\delta_{\alpha} \bar{C}\right)^{1 /(n+m)} & \text { if } \delta_{\alpha} \bar{C}<1,\end{cases}
\end{gathered}
$$

for some small prefixed positive real constant $\rho$ of an upper-bound specified later and

$$
\begin{gathered}
\bar{C}=\left\{C\left(\bar{\sigma}_{1}, \bar{\sigma}_{2}, \ldots, \bar{\sigma}_{n+m+1}\right):\left|C\left(\bar{\sigma}_{1}, \bar{\sigma}_{2}, \ldots, \bar{\sigma}_{n+m+1}\right)\right|\right. \\
\left.=\operatorname{Max}_{\sigma_{i} \in\{0,-1,1\}}\left|C\left(\sigma_{1}, \ldots, \sigma_{n+m+1}\right)\right|\right\}, \\
C\left(\sigma_{1}, \ldots, \sigma_{n+m+1}\right)=\sum_{k=1}^{n+m} \sum_{i_{1}, i_{2}, \ldots, i_{k}=1}^{n+m+1} \frac{1}{k !} \operatorname{Trace}\left(S_{\theta_{i_{1}}}\left(\theta_{0}\right) \tilde{S}_{\theta_{i_{1}} \ldots \theta_{i_{k}}}\left(\theta_{0}\right)\right) \prod_{j=i_{1}}^{i_{k}}\left[\sigma_{j}\right], \\
\left(\bar{\sigma}_{1}, \bar{\sigma}_{2}, \ldots, \bar{\sigma}_{n+m+1}\right)=\left\{\operatorname{Arg}\left(\sigma_{1}, \sigma_{2}, \ldots, \sigma_{n+m+1}\right): \bar{C}=C\left(\sigma_{1}, \sigma_{2}, \ldots, \sigma_{n+m+1}\right),\right. \\
\left.\sigma_{i} \in\{0,-1,1\}, i=1,2, \ldots, n+m+1\right\},
\end{gathered}
$$

where $\tilde{S}\left(\theta_{0}\right)$ is the matrix of cofactors of $S\left(\theta_{0}\right)$, with subscripts denoting partial firstor higher-order derivatives with respect to the respective arguments, and the first-order 
derivatives with respect to the parameter estimates are

$$
\begin{aligned}
& S_{a_{i}}\left(\theta_{0}\right)=\left.\frac{d S}{d a_{i}}\right|_{\theta_{0}} \\
& =\left[\begin{array}{ccc} 
& 0_{i \times(n+m)} & \\
\cdots & \cdots & \cdots \\
I_{n} & & 0_{m \times n} \\
\cdots & \cdots & \cdots \\
& 0_{(n-i) \times(n+m)} &
\end{array}\right] \longleftarrow(i+1) \text { th row }(i=1, \ldots, n), \\
& S_{b_{j}}\left(\theta_{0}\right)=\left.\frac{d S}{d b_{j}}\right|_{\theta_{0}} \\
& =\left[\begin{array}{ccc} 
& 0_{j \times(n+m)} & \\
\cdots & \cdots & \cdots \\
0_{n \times m} & & I_{n} \\
\cdots & \cdots & \cdots \\
& 0_{(m-j) \times(n+m)} &
\end{array}\right] \longleftarrow(j+1) \text { th row }(j=0,1, \ldots, m), \\
& \bar{\theta}=\theta+\bar{\delta} \\
& \bar{\delta}=[\delta \theta_{1}, \delta \theta_{2}, \ldots, \delta \theta_{n+m+1}, \overbrace{0, \ldots, 0}^{n}]^{T} \\
& =\left[\bar{\delta}_{0}^{T}, 0^{T}\right]^{T}=[\delta b_{0}, \delta b_{1}, \ldots, \delta b_{m}, \delta a_{1}, \delta a_{2}, \ldots, \delta a_{n}, \overbrace{0, \ldots, 0}^{n}]^{T}, \\
& \bar{a}_{i}=a_{i}+\delta a_{i}=a_{i}+\alpha \bar{\sigma}_{i}, \quad \bar{b}_{j}=b_{j}+\delta b_{j}=b_{j}+\alpha \bar{\sigma}_{n+1+j}, \quad i=1,2, \ldots, n, j=0,1, \ldots, m .
\end{aligned}
$$

Note that $\left(\bar{\sigma}_{1}, \bar{\sigma}_{2}, \ldots, \bar{\sigma}_{n+m+1}\right)^{T}$ is not necessarily a unique vector, whose components take values in the set $\{1,0,-1\}$ which maximizes the function $C\left(\sigma_{1}, \sigma_{2}, \ldots, \sigma_{n+m+1}\right)$ for the constraints $\sigma_{i} \in\{1,0,-1\}$ for $i=1,2, \ldots, n+m+1$. Assume that each $i$ th parameter component of $\theta_{0}$ is modified by an additive increment $\alpha \sigma_{i}$ so that the modification scheme is $\bar{\theta}_{0}=\theta_{0}+\alpha\left(\sigma_{1}, \ldots, \sigma_{n+m+1}\right)^{T}$. A well-known equation from linear algebra is

$$
\left.\frac{d \operatorname{Det}\left(S\left(\theta_{0}\right)\right)}{d \theta_{0 i}}\right|_{\theta_{0}=q_{0}}=\operatorname{Trace}\left(S_{\theta_{0 i}}\left(q_{0}\right) \cdot \tilde{S}\left(q_{0}\right)\right) \text {, }
$$

(see [9]) from which higher-order derivatives with respect to the various parameter vector components might also be calculated. Thus, by using a series Taylor expansion of the analytic multivariable function of the modified estimates $\operatorname{Det}\left(S\left(\bar{\theta}_{0,1}, \ldots, \bar{\theta}_{0, n+m+1}\right)\right)$ around $\operatorname{Det}\left(S\left(\theta_{0,1, \ldots, n+m+1}\right)\right.$ ) (later denoted by $\operatorname{Det}\left(S\left(\theta_{0}\right)\right)$ for notation simplicity purposes) which is considered as a multivariable function of all the parameter components, we have the identity $\operatorname{Det}\left(S\left(\bar{\theta}_{0}\right)\right)=\operatorname{Det}\left(S\left(\theta_{0}\right)\right)+C \alpha$, with the function $C$ being calculated from $(2.9 \mathrm{e})$. The switches in $h(t)$ between $\rho$ and $\rho^{\prime}$ given by (2.9a) have an objective of avoiding chattering so that the existence of solution is ensured for all time. Chattering could potentially 
arise if the Sylvester determinant would converge to a constant function $h$ while, at the same time, its time-derivative converges to zero with a change of sign. This phenomenon is avoided in this approach by using the switching rule (2.9a) by taking advantage of the fact that the unmodified and modified parameter estimates converge asymptotically to finite limits. Thus, if the Sylvester determinant converges to $\rho$ (or $\rho^{\prime}$ ) after a large but finite time, it cannot converge to $\rho^{\prime}$ (or $\rho$ ) while it remains in a certain small neighborhood centered at $\rho^{\prime}$ (or $\rho$ ). The avoidance of chattering guarantees the existence of solution. These features will be proved in the following section.

The above modification procedure basically operates as follows. Assume that $\theta_{i}$ is any estimate $a_{(\cdot)}$ or $b_{(\cdot)}$. If $\sigma=0$, then such a parameter does not contribute to the maximum $C$ (i.e., to $\bar{C}$ ). That means that if the parameter was accounted for in (2.9c) for eventual parameter modification with both signs, that is, $\sigma= \pm 1$, then $C$ would have a less absolute value. If $\sigma= \pm 1$, then the parameter contributes to $\bar{C}$, that is, if it is accounted for to calculate $\bar{C}$ which reaches a larger absolute value than for any other possible combinations for accounting or not all the remaining parameter estimates. At the end of the modification procedure, all the estimates whose corresponding $\bar{\sigma}_{(\cdot)}$ is \pm 1 become modified, while those ones whose corresponding $\bar{\sigma}_{(\cdot)}$ is zero remain unmodified. The use of two distinct values $\rho$ and $\rho^{\prime}$ to deal with switches in the determinant test is just to avoid that the potential situation, of the determinant converging to one of those values, implies the nonexistence of solution in the closed-loop system. Therefore, an isolated discontinuity (the test for switching $h_{(\cdot)}$ from one value to the other in (2.9a)) ensures the existence of solution, and the problem of convergence of the determinant of the Sylvester matrix of the unmodified estimates to one of those values is avoided since in finite, but large, time, the determinant is close to its limit, since the estimates have a limit, as proved in Theorem 3.2, and the corresponding discontinuity of $f$ ensures how new switches would arise. It is proved in the appendix, as an intermediate step in the proof of the subsequent controllability result, that $\bar{C} \neq 0$ for all time because not all the derivatives in (2.9e) with respect to the estimates evaluated at the parameter vector estimated from the algorithm (2.8) are zero. This feature makes possible that the Sylvester determinant of the modified estimates can always be modified with respect to its value prior to modification. It becomes obvious from the above modification philosophy that $|\bar{C}|$ can be replaced by any value of $|C|$ which is bounded from below by a positive constant. The main idea behind its proof is that the scalar function $\operatorname{Det}\left(S\left(\theta_{0}\right)\right)$ whose $n+m$ arguments are all the estimates $a_{(\cdot)}$ and $b_{(\cdot)}$ built through (2.9)-(2.10) is not constant at any real interval. This property will follow from the fact that at least one of its first-order derivatives (i.e., the components of its gradient with respect to the estimated parameters) or of its successive higher-order derivatives in the parameter space of estimates is nonzero. Therefore, the modification rule (2.9)-(2.10) allows the modification of the estimates when necessary so that the constraint $\left|\operatorname{Det}\left(S\left(\bar{\theta}_{0}\right)\right)\right| \geq \rho$ is fulfilled. The following result relies on the controllability of the modified estimated model.

Proposition 2.1. Assume that $\rho<|\sigma| /(6(n+m))$, where $(-\sigma)$ is the convergence abscissa of $C^{*}(D)$, that is, $0>-\sigma \geq-\operatorname{Max}_{1 \leq i \leq n}\left(\operatorname{Re}\left(-z_{i}\right)\right)$, where $z_{i}$ are the zeroes of the $C^{*}(D)$ polynomial (if they are not all simple, then the above second inequality is strict). Thus, the modified estimation scheme (2.9)-(2.10) of the plant model estimated from (2.8) is fulfilled 
at all time $\left|\operatorname{Det}\left(S\left(\bar{\theta}_{0}\right)\right)\right| \geq \rho>0$ so that such a model is controllable. Furthermore, there is no chattering caused by switches in the estimates modification rule (2.9a)-(2.9c).

2.3. Alternative modification of the estimation. A second variation of the above estimation modification rule of (2.9)-(2.10) is given below by modifying the algorithm rules (2.9). It is based on ensuring that the Sylvester matrix of the modified estimates is diagonally dominant in the case when that associated with estimates without modification is not guaranteed to be controllable under a sufficiency test. Such a test is based on the evaluation of matrix norms of $S\left(\theta_{0}\right)$ and it does not require the computation of its eigenvalues. First, define small positive real constants $\varepsilon_{b i}$, $\varepsilon_{0 i}$, and $\varepsilon_{0 i}^{\prime}$ fulfilling $\varepsilon_{b 2} \geq \varepsilon_{b 1}+\bar{\varepsilon}_{0}$, $\varepsilon_{02} \geq \varepsilon_{01}+\bar{\varepsilon}_{1}$, and $\varepsilon_{02}^{\prime} \geq \varepsilon_{01}^{\prime}+\bar{\varepsilon}_{1}^{\prime}$ as well as an arbitrary large real constant $T>0$ and an arbitrary large integer $N>0$. Then, establish Condition C. 1 for controllability test purposes of the estimated model before modification at any time $t$ as follows.

It is said that Condition C.1 holds at time $t$ if

$$
(n+m)^{1 / 2}\left\|S\left(\theta_{0}\right)\right\|_{\infty} \geq \frac{1}{\varepsilon_{0}^{\prime}}, \quad(n+m)^{-1 / 2}\left\|S\left(\theta_{0}\right)\right\|_{1} \leq \frac{1}{\varepsilon_{0}}
$$

with $\varepsilon_{0}(t)=\varepsilon_{0}(t-T)=\varepsilon_{0 i} ; \varepsilon_{0}^{\prime}(t)=\varepsilon_{0}^{\prime}(t-T)=\varepsilon_{0 i}^{\prime}$ for some $i \in\{1,2\}$ if $N_{t}^{-} \leq N ; \varepsilon_{0}(t)=$ $\varepsilon_{0 j} ; \varepsilon_{0}^{\prime}(t)=\varepsilon_{0 j}^{\prime}$ for some $j \in\{1,2\}$ with $j \neq i$ if $N_{t}^{-}=N+1$, where $N_{t}^{-}$is the overall number of times where inequalities (2.12a) are simultaneously violated with the same values for the constants on the finite, but large, time interval $[t-T, t)$, that is, with either $\left(\varepsilon_{01} ; \varepsilon_{01}^{\prime}\right)$ or $\left(\varepsilon_{02} ; \varepsilon_{02}^{\prime}\right)$. After any switch in the values of both constants in $(2.12 \mathrm{a}), N_{t}$ is set equal to zero; that is, if $N_{t}^{-}=N+1$, then $N_{t}=0$.

Also, Condition C.2 is now established for testing if $b_{m}$ belongs to a small neighborhood around zero as follows.

It is said that Condition C.2 holds at time $t$ if $\left|b_{m}\right| \geq \varepsilon_{b}$ with $\varepsilon_{b}(t)=\varepsilon_{b}(t-T)=\varepsilon_{b i}$ for some $i \in\{1,2\}$ if ${N^{\prime}}_{t}^{-} \leq N$; and $\varepsilon_{b}(t)=\varepsilon_{b j}$ for some $j \in\{1,2\}$ with $j \neq i$ if ${N^{\prime}}_{t}^{-}=N+1$, where ${N^{\prime}}_{t}^{-}$is the overall set of consecutive violations of Condition C. 2 on the time interval $[t-T, t)$, which operates in the same way as $N_{t}^{-}$for Condition C.1.

The parameter estimates are now modified as follows by using Conditions C.1 and C.2. Modify (2.10c) as follows:

$$
\begin{aligned}
& \delta a_{i}=\left\{\begin{array}{ll}
0 & \text { if Condition C.1 holds, } \\
-\alpha a_{i} & \text { otherwise, }
\end{array} \quad(i=0,1, \ldots, n, j=0,1, \ldots, m-1),\right. \\
& \delta b_{j}= \begin{cases}0 & \text { if Condition C.1 holds, } \\
-\alpha b_{j} & \text { otherwise, }\end{cases} \\
& \delta b_{m}= \begin{cases}0 & \text { if Condition C. } 1 \text { holds, } \\
\beta b_{m} & \text { if Condition C.1 does not hold and Condition C.2 holds, } \\
\beta^{\prime} & \text { if Conditions C.1 and C.2 do not hold, }\end{cases}
\end{aligned}
$$


where

$$
\beta=\left\{\begin{array}{c}
\alpha=\frac{\sum_{i=1}^{n}\left|a_{i}\right|+\sum_{i=0}^{m}\left|b_{i}\right|+\rho_{\alpha}-1}{\sum_{i=1}^{n}\left|a_{i}\right|+\sum_{i=0}^{m}\left|b_{i}\right|}, \\
\quad \text { if } \varepsilon_{b} \leq\left|b_{m}\right| \leq \sum_{i=1}^{n}\left|a_{i}\right|+\sum_{i=1}^{m-1}\left|b_{i}\right|+\gamma\left|b_{0}\right|+\rho_{\beta}, \\
\quad \text { if }\left|b_{m}\right| \geq \operatorname{Max}\left(\varepsilon_{b}, \sum_{i=1}^{n}\left|a_{i}\right|+\sum_{i=1}^{m-1}\left|b_{i}\right|+\gamma\left|b_{0}\right|+\rho_{\beta}\right), \\
\beta^{\prime}=\sum_{i=1}^{m-1}\left|a_{i}\right|+\sum_{i=1}^{m-1}\left|b_{i}\right|+\gamma\left|b_{0}\right|+\rho_{\beta}^{\prime}+\left|b_{m}\right|, \quad \gamma= \begin{cases}0 & \text { if } m=n, \\
1 & \text { if } m<n,\end{cases}
\end{array}\right.
$$

for prefixed given constants $\rho_{\alpha} \in\left(\rho_{\alpha}^{\prime}, 1\right], \rho_{\alpha}^{\prime} \in(0,1), \rho_{\beta}>0, \rho_{\beta}^{\prime}>0$, and $\beta$ and $\beta^{\prime}$ being calculated for all time for the implementation of the modification with $\alpha=\rho_{\alpha}=1$ if $\sum_{i=1}^{n}\left|a_{i}\right|+\sum_{i=0}^{m}\left|b_{i}\right|=0$.

Remark 2.2. Condition C.1 guarantees that all the absolute values eigenvalues of the Sylvester matrix of the estimated model (2.8) are positive and upper-bounded by a finite constant. As a result, Condition C.1 guarantees that $\left|\operatorname{Det}\left(S\left(\theta_{0}\right)\right)\right|$ is bounded away from zero. If it is violated, Condition C. 2 guarantees that the Sylvester matrix is diagonally dominant and then nonsingular. The scheme is stated in terms of achieving similar absolute relative increments in the modified estimated model for each nonzero estimate distinct of $b_{m}$. This is a major difference from the modification scheme (2.10)(2.12). The reason for using pairs of distinct test values for checking those conditions is to avoid chattering at their switching points, that is, when $\left\|S\left(\theta_{0}\right)\right\|_{\infty} \rightarrow 1 / \varepsilon_{0}^{\prime} \sqrt{n+m}$ and $\left\|S\left(\theta_{0}\right)\right\|_{1} \rightarrow \sqrt{n+m} / \varepsilon_{0}$ simultaneously as time tends to infinity with either constant values $\left(\varepsilon_{01} ; \varepsilon_{01}^{\prime}\right)$ and $\left(\varepsilon_{02} ; \varepsilon_{02}^{\prime}\right)$ (Condition C.1), or when $\left|b_{m}\right| \rightarrow \varepsilon_{b 1}\left(\varepsilon_{b 2}\right)$ (Condition C.2). The reason is that the unmodified estimates have finite limits depending on the initial conditions of the estimation algorithm so that each norm of the Sylvester matrix or $\left|b_{m}\right|$ cannot converge to two distinct values. A possible convergence to any of the switching points of the matrix norms and $\left|b_{m}\right|$ (which would imply chattering) is avoided with the use of Conditions C.1 and C.2 in (2.12). The mechanism used is to switch the values of the constants after a large number $N$ of consecutive switches have occurred with the same values of those constants over a prefixed arbitrarily large time interval $T$.

Remark 2.3. Note that the switches in the alternative modification scheme, (2.10a)(2.10b) and (2.12)-(2.13), automatically end in some finite time as it follows from the subsequent reasoning. Assume that the limits of the above norms and $\left|b_{m}\right|$ estimate are arbitrarily close to any of the switching points of Conditions C.1 and C.2 after a large time because the unmodified estimates are very close to their limit points. The existence of these limits will follow rigorously from the properties of the estimation and modification algorithms proved in the subsequent section. Thus, the switching conditions change 
after extra finite time to their alternative values because of the structure of the modification rule. More switches cannot occur after extra time $v T$ (some $v$ finite) since the (very close to their limits) unmodified estimates do not generate switches from Conditions C.1

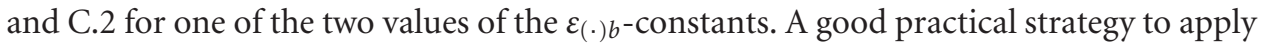
coherently Condition C.1 is the use of very large values for $\varepsilon_{0 i}^{\prime}$, very small ones for $\varepsilon_{0 i}$, and sufficient (although small) values for $\left|\varepsilon_{02}^{\prime}-\varepsilon_{01}^{\prime}\right|$ and $\left|\varepsilon_{02}-\varepsilon_{01}\right|$ for a fast ending of the switches of the modification mechanism. As in the basic modification mechanism, a possibly existing a priori knowledge on the true plant parameters could be used to design the various constants so that Conditions C. 1 and C. 2 hold for the true plant so that if the estimates converge to the true parameters, the modification mechanism is switched off automatically in finite time. However, the absence of that knowledge does not affect the stability of the closed-loop system.

The subsequent result is also proved in the appendix.

Proposition 2.4. If Condition C.1 holds, then the estimated plant model obtained from the algorithm (2.8) is controllable and its associate Sylvester matrix is nonsingular. If it does not hold, then the alternative modification scheme (2.12)-(2.13) is controllable for all time and it does not exhibit chattering generated by switches related to Conditions C.1 and C.2.

Remark 2.5. A simple motivation of Propositions 2.1 and 2.4 can be obtained from the perturbation Banach's lemma from Numerical Analysis [20] that establishes that small perturbations of nonsingular matrices yield nonsingular matrices. In terms of Sylvester matrices, the modification rule (2.10a) implies that $S^{\prime}\left(\bar{\theta}_{0}, \sigma_{(\cdot)}\right)=S^{\prime}\left(\theta_{0}\right)+\alpha \cdot \delta S^{\prime}\left(\theta_{0}, \sigma_{(\cdot)}\right)$, when the modification takes place, where the superscript prime indicates that the first rows and columns of the Sylvester matrices have been deleted, since they are irrelevant for their determinants and

$$
\delta S^{\prime}\left(\theta_{0}, \sigma_{(\cdot)}\right)=\left[\begin{array}{ccccccc}
\sigma_{1} & & & \vdots & \sigma_{0} & & 0 \\
\vdots & \sigma_{1} & & 0 & \sigma_{1} & & \vdots \\
\sigma_{n} & \vdots & \ddots & & \vdots & \ddots & \sigma_{0} \\
0 & \sigma_{n} & & \sigma_{1} & \sigma_{m} & & \sigma_{1} \\
\vdots & 0 & \ddots & \vdots & 0 & \ddots & \vdots \\
0 & \cdots & & \sigma_{n} & 0 & \cdots & \sigma_{m}
\end{array}\right]
$$

is an $(n+m)$-square matrix with each $\sigma_{i}$ potentially taking values in the set $\{0,-1,1\}$ of the modification scheme (2.9)-(2.10). By simple inspection, it is easy to see that $\delta S^{\prime}\left(\theta_{0}, \sigma_{(\cdot)}\right)$ can be built as being nonsingular for many of the choices of the $\sigma_{(\cdot)}$. (Constructions such as $\sigma_{n+i+1}=\sigma_{i}(i=1,2, \ldots, n)$ for $m=n-1$ have to be excluded since $\delta S^{\prime}\left(\theta_{0}, \sigma_{(\cdot)}\right)$ becomes singular.) Thus, $S^{\prime}\left(\bar{\theta}_{0}, \sigma_{(\cdot)}\right)$ is nonsingular and

$$
\left\|S^{-1}\left(\bar{\theta}_{0}, \sigma_{(\cdot)}\right)\right\| \leq \frac{\alpha^{-1}\left\|\delta S^{\prime-1}\left(\theta_{0}, \sigma_{(\cdot)}\right)\right\|}{1-\alpha^{-1}\left\|\delta S^{\prime-1}\left(\theta_{0}, \sigma_{(\cdot)}\right)\right\|\left\|S\left(\theta_{0}\right)\right\|}
$$


for any matrix norm provided that $\alpha>\left\|\delta S^{\prime-1}\left(\theta_{0}, \sigma_{(\cdot)}\right)\right\|\left\|S\left(\theta_{0}\right)\right\|$ is what follows if $\alpha>$ $(n+m+1) \operatorname{Max}\left(\sum_{i=1}^{n} a_{i}^{2}, \sum_{i=0}^{m} b_{i}^{2}\right)^{1 / 2}$ by taking $\ell_{1}$ matrix norms since $\delta_{\alpha} \geq \alpha$ from (2.9b)(2.9c). That means that if $\alpha$ or $\delta_{\alpha}$ is sufficiently large compared to a measure of the absolute values of the estimates, then the modified Sylvester matrix can be made nonsingular even if that prior to the modification is singular. A lower-bound for $\delta_{\alpha}$ is given explicitly in the proof of Proposition 2.1. The modification rule (2.12)-(2.13) is based on guaranteeing that either the unmodified Sylvester matrix is nonsingular and no modification is made or the modified Sylvester matrix is diagonally dominant and then nonsingular. For this case, $\delta S^{\prime}\left(\theta_{0}\right)=\operatorname{Diag}(-\alpha, \ldots,-\alpha, \beta) S^{\prime}\left(\theta_{0}\right)$ under modification for nonzero $b_{m}$ and $\delta S^{\prime}\left(\theta_{0}\right)=\operatorname{Diag}(-\alpha, \ldots,-\alpha, 0) S^{\prime}\left(\theta_{0}\right)+\beta^{\prime}$ otherwise.

2.4. Stabilizing adaptive control law. Introducing (2.10a) into (2.8a), we obtain

$$
\begin{aligned}
D^{n} y_{f} & =e+\theta^{T} \varphi=e+\left(\bar{\theta}^{T}-\bar{\delta}^{T}\right) \varphi \\
& =e+A(D, t) y_{f}+B(D, t) u_{f}+\varepsilon_{0}^{T}(t) i_{\varphi}(t)
\end{aligned}
$$

with $A(D, t)$ and $B(D, t)$ being time-varying polynomials associated with the estimates obtained from (2.8), which define the estimated model of the plant prior to eventual modification, and whose adjustable parameters are the components of the a priori estimated vector $\theta$. The filtered and unfiltered control inputs are generated from the adaptive version of (2.4)-(2.5),

$$
\begin{gathered}
S(D, t) u_{f}=-R(D, t) y_{f}, \\
u=\left(E^{*}(D)-S(D, t)\right) u_{f}-R(D, t) y_{f},
\end{gathered}
$$

so that the following closed-loop Diophantine equation is satisfied by the controller polynomials $R(D)$ and $S(D)$ which are calculated from modified parameter estimates

$$
\bar{A}(D, t) S(D, t)+\bar{B}(D, t) R(D, t)=C^{*}(D)
$$

with $\bar{A}(D, t)=A(D, t)+\delta A(D, t), \bar{B}(D, t)=B(D, t)+\delta B(D, t), \delta A(D, t)=\sum_{i=1}^{n} \delta a_{i} D^{n-i}$, and $\delta B(D, t)=\sum_{i=0}^{m} \delta b_{i} D^{m-i}$. The solution is unique since the modified plant parameter estimated model is controllable at all time, which implies that the time-varying polynomials $\bar{A}(D, t)$ and $\bar{B}(D, t)$ are coprime for all time [5] in the sense that the absolute value of the Sylvester matrix of the modified plant estimates is nonzero and lower-bounded by a positive real constant. This makes the control law nonsingular and then solvable since the Diophantine equation (2.19a) built with the modified plant estimates, which is used for the controller synthesis, is nonsingular.

2.5. Calculation of the parameters of the adaptive stabilizer. The expression (2.19a) is equivalent to the following algebraic linear system:

$$
S\left(\bar{\theta}_{0}\right) v=c^{*}
$$


for all time with

$$
v=\left[1, s_{1}, \ldots, s_{n}, r_{0}, r_{1}, \ldots, r_{m-1}\right]^{T}, \quad c^{*}=\left[1, c_{1}^{*}, c_{2}^{*}, \ldots, c_{n^{*}}^{*}\right]^{T},
$$

which is uniquely solvable with updated parameters at all time in $s_{(\cdot)}$ and $r_{(\cdot)}$ which are used to generate the filtered plant input (2.4) so that the reference closed-loop dynamics characteristic equation is $C^{*}(D)=0$.

\section{Stability results}

The following assumption on some of the design constants is introduced to guarantee the stability of the closed-loop system under estimates modification.

Assumption 3.1. (a) The design constant $\rho$ in (2.9a) is chosen sufficiently small according to the constraint $\rho<|\sigma| / 6(n+m)$ in the basic modification scheme of Section 2.2, (2.9)(2.10).

(b) The design constant $\varepsilon_{01}^{\prime}$ is sufficiently large and the design constants $\varepsilon_{02}, \varepsilon_{b 2}$, and $\rho_{b}^{\prime}$ are sufficiently small so that $|\sigma|>\operatorname{Max}\left(\bar{\delta}_{1}^{\prime}, \bar{\delta}_{2}^{\prime}\right)$ with $0<\varepsilon_{01}+\bar{\varepsilon}_{0} \leq \varepsilon_{02}<\sqrt{n+m}$, where

$$
\begin{gathered}
\bar{\delta}_{1}^{\prime}=\left(1-\rho_{\alpha}\right)+\sqrt{2}\left(1+\frac{1}{\varepsilon_{01}^{\prime} \sqrt{n+m}}+\rho_{\beta}^{\prime}+\varepsilon_{b 2}\right), \\
\bar{\delta}_{2}^{\prime}=\left(2+\frac{\varepsilon_{02}}{\sqrt{n+m}-\varepsilon_{02}}\right)\left(1-\rho_{\alpha}\right),
\end{gathered}
$$

in the implementation of the alternative modification scheme of Section 2.4, (2.10a)(2.10b)-(2.13).

Theorem 3.2. The adaptive control law (2.17)-(2.18), under the estimation scheme (2.8)(2.10) (or (2.8), (2.10a), and (2.12)-(2.13)) and (2.19), has the following properties when applied to the plant (2.1) provided that Assumption 3.1 holds:

(i) $\theta, \bar{\theta}$, and $P$ are uniformly bounded and the modified estimated plant model is controllable at all time;

(ii) $e$ and $P \varphi$ are in $L_{2}$;

(iii) $\theta, P, \bar{\theta}, s_{i}$, and $r_{j}(i=1,2, \ldots, n, j=0,1, \ldots, m-1)$ converge asymptotically to finite limits for any bounded initial conditions for the plant and the estimation algorithm; also, the Sylvester determinants of the unmodified and modified parameter estimates converge asymptotically to finite limits;

(iv) $D^{i} u_{f}, D^{i} y_{f}(i=0,1, \ldots, n-1)$ and $u$ and $y$ are uniformly bounded and converge asymptotically to zero.

Note that $e \in L_{2} \cap L_{\infty}$ from Theorem 3.2(i) and (iv) so that $e \rightarrow 0$ as $t \rightarrow \infty$ and $\theta \in L_{\infty}$, and converges to a finite limit. Also, $\|\dot{\tilde{\theta}}\| \in L_{\infty}$ from $(2.8 \mathrm{~b})$ since $P \in L_{\infty}$ and $\varphi \in L_{\infty}$. These properties guarantee that $\operatorname{Det}\left(S\left(\theta_{0}\right)\right)$ and $\theta_{0}$ are bounded and converge to finite limits so that the modification $\bar{\delta}$ is bounded and converges for both proposed modification schemes (2.9)-(2.10) and (2.10a), (2.12)-(2.13). 
Remark 3.3. Assume that the plant is not perfectly modeled and/or it is subject to bounded disturbances with the unmodeled dynamics being related to $u_{f}$ by a exponentially stable transfer function. Thus, it is modeled after filtering as $A^{*}(D) y_{f}=B^{*}(D) u_{f}+$ $\eta_{f}+\varepsilon_{0}^{T}(t) i_{\varphi}(t)$ with $\eta_{f}=\left(1 / E^{*}(D)\right) \eta(t)$. Assume that (2.1) is controllable when $\eta \equiv 0$ and that there exists an overbounding measurable function

$$
\bar{\eta}_{f}(t)=\varepsilon_{1} \rho(t)+\varepsilon_{2}=\varepsilon_{1} \operatorname{Sup}_{0 \leq \tau \leq t}\left\{\left\|\varphi(\tau) e^{-\sigma_{0}(t-\tau)}\right\|\right\}+\varepsilon_{2} \geq\left|\eta_{f}\right|
$$

for some nonnegative real constants $\varepsilon_{i}(i=1,2)$, where $v(t)$ is a vector whose components are $D^{j} u_{f}$ and $D^{j} u_{f}, j=0,1, \ldots, n-1$ (see $[4,5,16]$ ). The estimation scheme of (2.8) is modified by premultiplying the right-hand sides of $(2.8 \mathrm{a})-(2.8 \mathrm{~b})$ by the normalizing factor $b:=g s /\left(1+\gamma \phi^{T} P \phi\right)$, where

$$
\begin{aligned}
s: & = \begin{cases}0 & \text { if } t \in \mathbf{I}_{1}:=\left\{t \in \mathbb{R}_{0}^{+}:|e|<\mu \bar{\eta}_{f}\right\}, \\
\frac{f\left(\mu \bar{\eta}_{f}, e\right)}{e} & \text { otherwise (i.e., for } \left.t \in \mathbf{I}_{2}:=\mathbb{R}_{0}^{+}-\mathbf{I}_{1}\right),\end{cases} \\
f(\sigma, e): & = \begin{cases}e-\sigma & \text { if } e>\sigma, \\
0 & \text { if }|e| \leq \sigma, \\
e+\sigma & \text { if } e<-\sigma,\end{cases}
\end{aligned}
$$

with $g, \gamma, \mu>1$ prefixed positive constants. Note that $b(t)$ includes a relative dead zone for a small prediction error related to the size of the unmodeled dynamics (see, e.g., $[4,5,16])$. Thus, it can be proved that $\theta \in L_{\infty}, \bar{\theta} \in L_{\infty}$, and both converge asymptotically to finite limits as time tends to infinity, $P \varphi \in L_{2}$ and $b\left|\eta_{f}^{2}-e^{2}\right| \in L_{1} \cap L_{\infty}$, and also that the filtered and unfiltered input and output signals are uniformly bounded. The proof is very similar to that of Theorem 3.2 and it is then omitted. The basic mathematical tool used for this proof is to prove that a Lyapunov-like candidate $V(t)=\tilde{\theta}^{T}(t) P(t) \tilde{\theta}(t)$ is finitely upper-bounded for any bounded initial a priori (unmodified) parametrical errors since it has a nonpositive time-derivative for all time, while it remains constant over $\mathbf{I}_{1}$ and converges to a finite limit as time tends to infinity. This ensures the uniform boundedness of all the a priori estimates and associated parametrical errors and their convergence to finite limits as time tends to infinity. From the modification rule, it follows that the modified estimates exhibit similar properties. It is also proved easily that the Diophantine equation used for the controller synthesis based on the use of the modified estimates is nonsingular, and then solvable from the modification rule, since the modified estimation model is controllable for all time.

\section{Numerical example}

A numerical example is now tested for a nominally and inversely unstable plant (2.1) parametrized by $A^{*}(D)=D^{4}+0.75 D^{3}+0.5 D^{2}+0.25 D+0.25$ and $B^{*}(D)=0.75 D^{3}+$ $(2 / 3) D^{2}+0.25 D+0.25$ with initial conditions $(-5,-7,0,0)^{T}$ with filter parameter $E^{*}(D)=(D+6.93)^{2}$. The estimation algorithm used prior to modification is that 

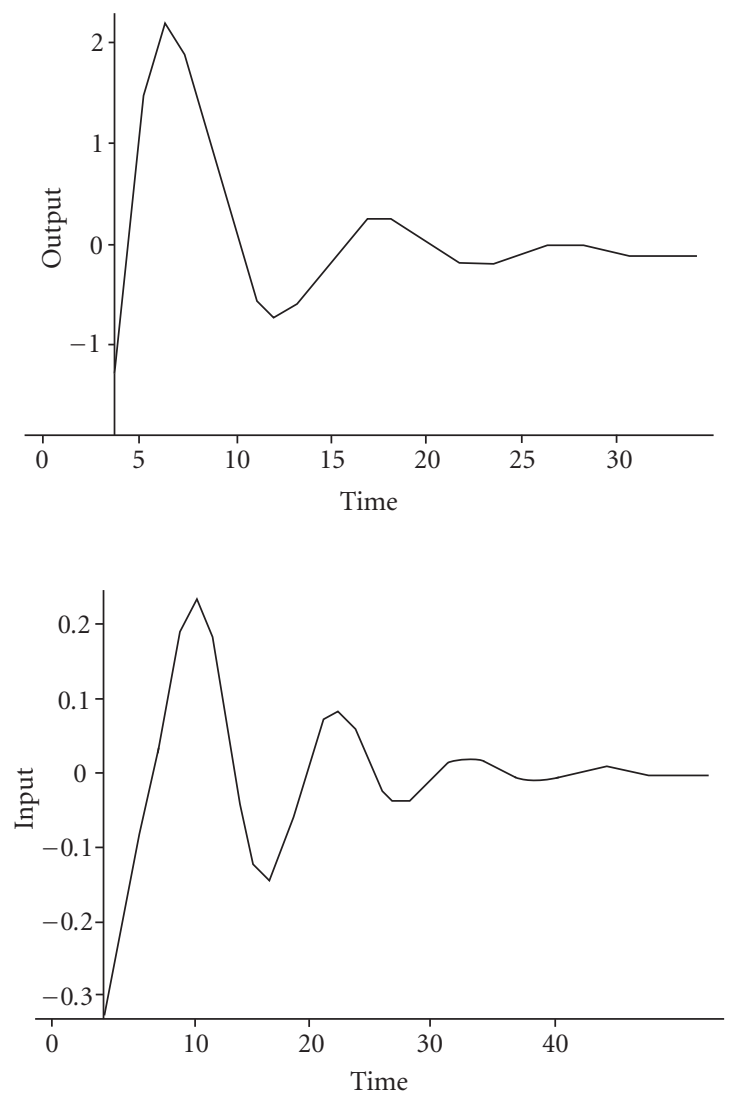

Figure 4.1. Output and input versus time of the closed-loop system.

of Remark 2.3. The unmodeled dynamics is defined by a second-order differential equation $\eta+0.12 \eta-7.8=7.8 u$. The estimation modification algorithm used is that of (2.8)(2.10) with the replacement of (2.9)-(2.10) by (2.12)-(2.13). The determinant threshold for parameter modification of the estimates is $\rho=0.01$. The adaptive stabilizer satisfies the constraints $\operatorname{deg}(R(D))=\operatorname{deg}(S(D))-1=1$. The initialization of the estimation algorithm is $b_{0}(0)=1, b_{1}(0)=-0.008, b_{2}(0)=-0.003, a_{1}(0)=0.005, a_{2}(0)=-0.005$, $a_{3}(0)=0, a_{4}(0)=0$. The parameter $b_{3}^{*}$ is assumed to be known and then deleted from the estimation algorithm. The estimates of the initial conditions of the plant (2.1) are zero. The covariance matrix is initialized to $P(0)=\operatorname{Diag}\left(10^{6}\right)$ and $g=\gamma=1, \mu=1.04$. The absolute overbounding of the unmodeled dynamics contribution is computed with constants $\varepsilon_{1}=1, \varepsilon_{2}=10^{-5}$, and $\sigma_{0}=0.1$. The output and input versus time are shown on Figure 4.1. Figure 4.2 shows the absolute value of the Sylvester determinant related to the estimates and modified estimates, respectively. Note, in particular, that the absolute value of the modified Sylvester determinant exceeds a positive real constant which ensures the (strict) coprimeness of the modified estimated plant and then the nonsingularity of the 

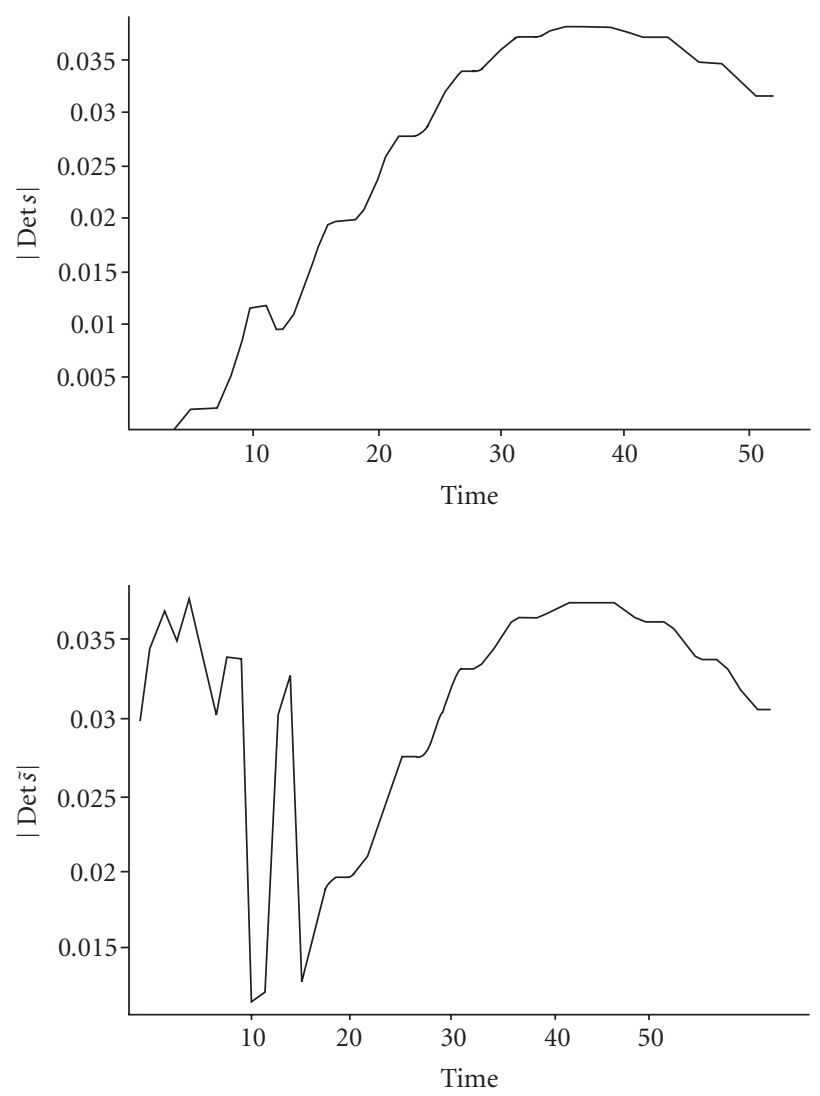

Figure 4.2. Absolute Sylvester determinants of the a priori and modified estimation schemes.

adaptive control law avoiding chattering and ensuring the boundedness of the input and output signals so that the closed-loop stability is guaranteed.

\section{Conclusions}

An adaptive stabilizer possibly possessing an unstable inverse has been proposed for a continuous-time plant without assuming the inverse stability of the plant, a priori knowledge on the plant parameters and knowledge of the high-frequency gain sign. The adaptive stabilizer is of pole/placement-based type. It basically consists of an estimation algorithm with covariance matrix adaptation with a subsequent parameter estimation modification of the parameter estimates constructed in such a way that the Diophantine equation used for the controller synthesis from the modified plant estimates is nonsingular, since zero-pole cancellations are avoided, and then solvable for all time. Two modification schemes have been proposed, which ensures the controllability of the modified estimated plant model. The closed-loop system is proved to be robustly stable against the presence of a wide class of unmodeled dynamics (possibly including bounded noise) with all the 
relevant signals in the loop remaining bounded for all time. A simulated example has corroborated the usefulness of the proposed scheme.

\section{Appendix}

Proof of Proposition 2.1. Firstly, note that the first-order derivatives of the determinant with respect to any parameter estimate are calculated from elementary algebra as follows (see, e.g., [9]):

$$
\frac{\partial}{\partial \theta_{i}} \operatorname{Det}\left(S\left(\theta_{0}\right)\right)=\operatorname{Trace}\left(\frac{\partial S\left(\theta_{0}\right)}{\partial \theta_{i}} \tilde{S}\left(\theta_{0}\right)\right)
$$

which holds when taking derivatives of determinants with respect to any value of the parameter estimate $\theta_{i}$ for $i=1,2, \ldots, n+m+1$. The derivatives are evaluated at $\theta_{0}$. However, it is clear from $(2.9 \mathrm{e})$ that

$$
S_{\theta_{i_{1}}, \ldots, \theta_{i_{k}}}=\frac{\partial^{k} S\left(\theta_{0}\right)}{\partial \theta_{i_{1}}^{k} \cdots \partial \theta_{i_{k}}^{k}}=0, \quad k=2,3, \ldots, n+m+1,
$$

with all the partial derivatives being evaluated at $\theta_{0}$. Also, since $\tilde{S}\left(\theta_{0}\right)$ is a matrix of cofactors, it contains products of at most $(n+m)$ parameters at each of its entries so that $\tilde{S}_{\theta_{i_{1}} \cdots \theta_{i_{k}}}\left(\theta_{0}\right)=0$ if $k>n+m$ for any integers $i_{j} \geq 1$ for $j=1,2, \ldots, k$. Now, $\operatorname{Det}\left(S\left(\bar{\theta}_{0}\right)\right)$ is expanded in Taylor series around $\operatorname{Det}\left(S\left(\theta_{0}\right)\right)$ by taking successive derivatives with respect to parameter components evaluated at $\theta_{0}$ by starting with (A.1) while zeroing any derivatives of an order higher than $(n+m)$. One obtains directly

$$
\operatorname{Det}\left(S\left(\bar{\theta}_{0}\right)\right)=\operatorname{Det}\left(S\left(\theta_{0}\right)\right)+\Delta\left(\theta_{0}, \bar{\theta}_{0}\right)
$$

with

$$
\Delta\left(\theta_{0}, \bar{\theta}_{0}\right)=\sum_{k=1}^{n+m} \sum_{i_{1}, i_{2}, \ldots, i_{k}=1}^{n+m+1} \frac{1}{k !} \operatorname{Trace}\left(S_{\theta_{i_{1}}}\left(\theta_{0}\right) \tilde{S}_{\theta_{i_{1}} \cdots \theta_{i_{k}}}\left(\theta_{0}\right)\right) \prod_{j=i_{1}}^{i_{k}}\left(\bar{\theta}_{j}-\theta_{j}\right)
$$

being the maximum absolute achievable increment between the modified and unmodified determinants. Now, it is proved by contradiction that

$$
\operatorname{Trace}\left(S_{\theta_{i_{1}}}\left(\theta_{0}\right) \tilde{S}_{\theta_{i_{1}} \ldots \theta_{i_{k}}}\left(\theta_{0}\right)\right)=0 \quad \forall i_{k} \in\{1, \ldots, n+m+1\}, k=1,2, \ldots, n+m,
$$

is impossible since (A.4) depends on the estimates of the plant parameters irrespective of the modification scheme. Now, assume that $\left|\operatorname{Det} S\left(\theta_{0}\right)\right| \neq \zeta<\rho$ with $\zeta>0$. Then, note from the definition of $S\left(\bar{\theta}_{0}\right)$ that $\left|\operatorname{Det}\left(S\left(\bar{\theta}_{0}\right)\right)\right|=\zeta$ with arbitrary nonzero $\zeta$ if the subsequent modification rule is used after estimation: $\delta a_{i}=-a_{i}, \delta b_{j}=-b_{j}$, and $\delta b_{m}=$ $\pm \zeta^{1 / n}-b_{m}$ for $i=1,2, \ldots, n, j=0,1, \ldots, m$. Assume that (A.4) holds. Thus, one has the impossible relationships $\zeta=\left|\operatorname{Det}\left(S\left(\bar{\theta}_{0}\right)\right)\right|=\left|\operatorname{Det}\left(S\left(\theta_{0}\right)\right)\right| \neq \zeta$ by using a Taylor series expansion in the parameter space of the modified estimates around the estimated ones obtained from (2.8) according to (A.3). Thus, (A.4) is false since all the derivatives used in (A.3) are not dependent on the modification scheme. Then, there is at least one parameter 
component $\theta_{i}$ of $\theta_{0}$ for which $\operatorname{Trace}\left(S_{\theta_{i_{1}}}\left(\theta_{0}\right) \tilde{S}_{\theta_{i_{1}} \cdots \theta_{i_{k}}}\left(\theta_{0}\right)\right) \neq 0$ and then $\bar{C}$ in $(2.9 \mathrm{~d})-(2.9 \mathrm{e})$ is nonzero. Thus, $\operatorname{Det}\left(S\left(\theta_{0}\right)\right)$ is not constant for all the values of the components of $\theta_{0}$ belonging to arbitrary real intervals, and a modification $\theta_{0} \rightarrow \bar{\theta}_{0}$ can be carried out to guarantee that $\left|\operatorname{Det}\left(S\left(\bar{\theta}_{0}\right)\right)\right| \geq \rho$. If $\delta_{\alpha}$ is discontinuous at $t$, then $\left|\delta_{\alpha}\left(t^{+}\right)\right| \geq 2 \rho /|\bar{C}|$ if $h\left(t^{+}\right)=\rho$ and $h\left(t^{-}\right)=\rho^{\prime}$, and $\left|\delta_{\alpha}\left(t^{+}\right)\right| \geq 2 \rho^{\prime} /|\bar{C}|$ if $h\left(t^{+}\right)=\rho^{\prime}$ and $h\left(t^{-}\right)=\rho$. In any of the above situations, $\alpha(t) \neq 0$. The switches in $h(t)$ make these eventual discontinuities occur only at isolated time instants. Direct calculations yield

$$
\begin{aligned}
\left|\operatorname{Det}\left(S\left(\bar{\theta}_{0}\right)\right)\right| & =\left|\operatorname{Det}\left(S\left(\theta_{0}\right)\right)+\Delta\left(\theta_{0}, \bar{\theta}_{0}\right)\right| \\
& \geq\left|\delta_{\alpha}\right||\bar{C}|-\left|\operatorname{Det}\left(S\left(\theta_{0}\right)\right)\right| \\
& \geq 3 \rho-\operatorname{Det}\left(S\left(\theta_{0}\right)\right) \operatorname{Sign}(\bar{C})-\left|\operatorname{Det}\left(S\left(\theta_{0}\right)\right)\right| \geq \rho>0 .
\end{aligned}
$$

Note that $\delta_{\alpha}=\alpha$ if $\delta_{\alpha} \geq 1$ (which implies that $\alpha^{j} \geq \alpha$ for $j>1$ ) and $\delta_{\alpha}=\alpha^{n+m}$ if $\delta_{\alpha}<1$ (which implies that $\alpha^{j}<\alpha$ for $j>1$ ) with $\delta_{\alpha}$ and $\alpha$ being chosen according to (2.9a)(2.9b). Such a constraint establishes the first inequality in (A.5) since $\left|\Delta\left(\theta_{0}, \bar{\theta}_{0}\right)\right| \geq\left|\delta_{\alpha} \bar{C}\right|$ by (A.3b). Thus, the first part of Proposition 2.1 has been proved. The absence of chattering follows directly since the $\alpha$-function is continuous at $\delta_{\alpha} \bar{C}=1$ since $\delta_{\alpha} \bar{C}=$ $\left[\left(\delta_{\alpha} \bar{C}\right)^{1 /(n+m)}\right]_{\delta_{\alpha} \bar{C}=1}$. The eventual discontinuities in the determinant test (2.9b) are isolated at any time, which is guaranteed by the switches in $h(t)$ given by $(2.9 \mathrm{a})$.

Proof of Proposition 2.4. One has from the definitions and properties of the $\ell_{1}, \ell_{2}$, and $\ell_{\infty}$ matrix norms (see, e.g., $[1,12]$ ),

$$
\begin{aligned}
(n+m)^{-1 / 2}\left\|S\left(\theta_{0}\right)\right\|_{2} & =(n+m)^{-1 / 2}\left|\lambda_{\max }^{1 / 2}\left(S^{T}\left(\theta_{0}\right) S\left(\theta_{0}\right)\right)\right| \\
& =(n+m)^{-1 / 2}\left|\frac{1}{\lambda_{\min }^{1 / 2}\left(S^{T}\left(\theta_{0}\right) S\left(\theta_{0}\right)^{-1}\right)}\right| \\
& \leq(n+m)^{1 / 2}|| S\left(\theta_{0}\right) \|_{1},
\end{aligned}
$$

where $\left|\lambda_{\max }(\cdot)\right|$ and $\left|\lambda_{\min }(\cdot)\right|$ denote, respectively, the maximum and minimum modules of the eigenvalues of the $(\cdot)$-matrix. Thus, the following two inequalities follow directly from (A.6):

$$
\begin{aligned}
\left|\lambda_{\min }^{1 / 2}\left(S^{T}\left(\theta_{0}\right) S\left(\theta_{0}\right)^{-1}\right)\right| & =\frac{1}{\left|\lambda_{\max }^{1 / 2}\left(S^{T}\left(\theta_{0}\right) S\left(\theta_{0}\right)\right)\right|} \\
& =\frac{1}{\left\|S\left(\theta_{0}\right)\right\|_{2}} \geq \frac{1}{(n+m)^{1 / 2}|| S\left(\theta_{0}\right) \|_{1}} \\
& =\frac{1}{(n+m)^{1 / 2} \operatorname{Max}\left(1+\sum_{i=1}^{n}\left|a_{i}\right|, \sum_{i=0}^{m}\left|b_{i}\right|\right)},
\end{aligned}
$$




$$
\begin{aligned}
\mid \lambda_{\max }^{1 / 2} & \left(S^{T}\left(\theta_{0}\right) S\left(\theta_{0}\right)^{-1}\right) \mid \\
& =\frac{1}{\left|\lambda_{\min }^{1 / 2}\left(S^{T}\left(\theta_{0}\right) S\left(\theta_{0}\right)\right)\right|} \leq \frac{1}{\left|\lambda_{\max }^{1 / 2}\left(S^{T}\left(\theta_{0}\right) S\left(\theta_{0}\right)\right)\right|} \\
& =\frac{1}{|| S\left(\theta_{0}\right) \|_{2}} \leq \frac{1}{(n+m)^{-1 / 2}|| S\left(\theta_{0}\right) \|_{\infty}} \\
& =\frac{1}{(n+m)^{-1 / 2}\left(\sum_{i=1}^{n-1}\left|a_{i}\right|+\sum_{i=1}^{m-1}\left|b_{i}\right|+\operatorname{Max}\left(1+\left|b_{0}\right|,\left|a_{n}\right|+\left|b_{m}\right|\right)\right)}
\end{aligned}
$$

which implies

$$
0<\frac{1}{\varepsilon_{0}^{\prime}} \leq\left|\lambda_{\min }^{1 / 2}\left(S^{T}\left(\theta_{0}\right) S\left(\theta_{0}\right)\right)\right| \leq\left|\lambda_{\max }^{1 / 2}\left(S^{T}\left(\theta_{0}\right) S\left(\theta_{0}\right)\right)\right| \leq \frac{1}{\varepsilon_{0}}<\infty
$$

if Condition C. 1 holds. Thus, Condition C.1 guarantees that $S\left(\theta_{0}\right)$ is nonsingular (i.e., the estimated plant model is controllable) and a parameter modification is not performed in (2.12)-(2.13). If Condition C.1 does not hold, then $S\left(\theta_{0}\right)$ is not guaranteed to be nonsingular according to the test of (A.8). Thus, the estimation modification procedure of (2.10a), (2.12)-(2.13), when Condition C.1 does not hold, guarantees that

$$
1>\sum_{i=1}^{n}\left|\bar{a}_{i}\right|+\sum_{i=0}^{m-1}\left|\bar{b}_{i}\right|, \quad\left|\bar{b}_{m}\right|>\sum_{i=1}^{n}\left|\bar{a}_{i}\right|+\sum_{i=1}^{m-1}\left|\bar{b}_{i}\right|+\gamma\left|\bar{b}_{0}\right| .
$$

Now, note that if (A.9) holds, then the modified $S\left(\bar{\theta}_{0}\right)$ is diagonally dominant (i.e., the algebraic sum of all the entries of any row vector has the sign of its component at the main diagonal), which follows directly by inspection from its definition since for such a matrix structure, it suffices to guarantee diagonal dominance for the $n$th and $(n+1)$ th rows. Since all diagonally dominant matrices are nonsingular [12], $S\left(\bar{\theta}_{0}\right)$ is nonsingular and the modified estimated plant model is controllable. The proof of nonsingularity has been completed.

The absence of chattering follows from the use of two possible values of all the $\varepsilon_{(\cdot)}$ constants in Conditions C. 1 and C.2, the fact that those values are modified after $N$ consecutive switches with the same values of the constants over finite intervals of length $T$, and the feature that the estimates prior to the modification have finite limits (see also Remarks 2.2 and 2.3).

Proof of Theorem 3.2. The subsequent proof applies for both modification schemes (2.9)(2.10) and (2.10a)-(2.10b), (2.12)-(2.13).

(i)-(ii) Note that $\dot{P}^{-1}=-P^{-1} \dot{P} P^{-1}=\varphi \varphi^{T}$ from (2.8c). Define the Lyapunov function candidate $V=\tilde{\theta}^{T} P^{-1} \tilde{\theta}$, where $\tilde{\theta}=\hat{\theta}-\theta^{*}$ is the parametrical error before the modification of the estimates. Thus, (2.8a) can be rewritten as $e=-\tilde{\theta}^{T} \varphi$ and $\dot{V}=-\left(\tilde{\theta}^{T} \varphi\right)^{2}=$ $-e^{2} \leq 0$ after direct calculations with $V$ and (2.8) [2]. Thus, $\int_{0}^{t} e^{2}(\tau) d \tau=V(0)-V(t) \leq$ $V(0)<\infty$ for all $t \geq 0$ so that $e \in L_{2}$ and $\infty>\tilde{\theta}^{T} P^{-1} \tilde{\theta} \geq \lambda_{\min }\left(P^{-1}\right) \tilde{\theta} \tilde{\theta}^{T}$, with $\lambda_{\min }\left(P^{-1}\right)$ being the minimum eigenvalue of $P^{-1}$ so that $\tilde{\theta}$ is uniformly bounded since the maximum 
eigenvalue of $P, \lambda_{\max }(P)$, is upper-bounded by a positive finite constant and then $\lambda_{\min }\left(P^{-1}\right)=\lambda_{\max }^{-1}(P)>0$ for all $t \geq 0$. Thus, $P \theta$ is uniformly bounded and $\|P\|,\|\theta\|$, and $\|\bar{\delta}\|$ are in $L_{\infty}$ from (2.10) since $\theta=\left(\theta_{0}^{T}, \varepsilon_{0}^{T}\right)^{T}$ and $\theta_{0}$ and $\operatorname{Det}\left(S\left(\theta_{0}\right)\right)$ are uniformly bounded for all $t \geq 0$. Thus, the modified parameter vector $\bar{\theta}=\left(\bar{\theta}_{0}^{T}, \varepsilon_{0}^{T}\right)^{T}$ is also uniformly bounded for all $t \geq 0$. The modified estimated plant model is controllable since $\infty>\left|\operatorname{Det}\left(S\left(\bar{\theta}_{0}\right)\right)\right| \geq \rho>0$ from (2.9)-(2.10) and the fact that $\bar{\theta}_{0}$ is uniformly bounded for all $t \geq 0$. On the other hand, $P \varphi \in L_{2}$ since $\operatorname{tr}(\dot{P})=-\|P \varphi\|_{2}^{2} \in L_{1}$ from (2.8c) with $\|\cdot\|_{2}$ denoting the spectral (or Euclidean) vector norm. Thus, (i) and (ii) have been proved.

(iii) It is standard to prove that $P$ and $\theta$ converge asymptotically as time tends to infinity from (2.8b), since $P(t)$ is positive-definite for all $t \geq 0$ (possibly being only positivedefinite at the limit as time tends to infinity) with negative semidefinite time-derivative (so that it is monotonically decreasing for all time), and the fact that $\lim _{t \rightarrow \infty}\left(\int_{0}^{t}\|\dot{\theta}\| d \tau\right) \leq$ $(1 / 2)\left[\lim _{t \rightarrow \infty}\left(\|P \varphi\|^{2} d \tau\right)+\lim _{t \rightarrow \infty}\left(\int_{0}^{t} e^{2} d \tau\right)\right]<\infty$ since $P \varphi \in L_{2}$ and $e \in L_{2}$, which implies that $\dot{\theta} \in L_{1}$ and the $\theta$ converges from (ii) (see [7]). Also, $\theta_{0}$ converges since $\theta$ converges, and thus $\operatorname{Det}\left(S\left(\theta_{0}\right)\right)$ converges to finite constant values as time tends to infinity. From the fact that $\theta_{0}$ converges, the possible switches in (2.9a)-(2.9b) end in finite time since there exists a large finite time $\bar{t}_{0}$ such that $\theta$ and $\operatorname{Det}\left(S\left(\theta_{0}\right)\right)$ are close to their limits and the piecewise-constant $h$-function maintains a constant value ( $\rho$ or $\rho^{\prime} \geq \rho$ ) for all time $t \geq \bar{t}_{0}$ (see (2.19a)-(2.19b)). As a result, $\alpha, \sigma_{(\cdot)}, \bar{\sigma}_{(\cdot)}$, and $\bar{C}$ converge. Thus, the modified parameter vector $\bar{\theta}$, and then $\operatorname{Det}\left(S\left(\bar{\theta}_{0}\right)\right)$, converge asymptotically to finite limits. As a result, each controller parameter, namely, each coefficient of $R(D, t)$ and $S(D, t)$, converges to a finite limit value and (iii) has been proved.

(iv) Note that a direct calculation from (2.16) yields for $m \leq \leq n-1$,

$$
D^{n} y_{f}=e+\left(\bar{\theta}^{T}-\bar{\delta}^{T}\right) \varphi=e+\sum_{i=0}^{m} \bar{b}_{i} D^{m-i} u_{f}-\sum_{i=1}^{n} \bar{a}_{i} D^{n-i} y_{f}-\bar{\delta}_{0}^{T} \varphi_{0}
$$

and the substitution of $D^{n} u_{f}$ obtained explicitly from (2.17) into (2.16) yields for $m=n$,

$$
\begin{aligned}
D^{n} y_{f}= & e-\bar{b}_{0}\left[\sum_{i=1}^{n} s_{i} D^{n-i} u_{f}+\sum_{i=0}^{n-1} r_{i} D^{n-i-1} y_{f}\right] \\
& +\left[\sum_{i=1}^{n} \bar{b}_{i} D^{n-i} u_{f}-\sum_{i=0}^{n-1} \bar{a}_{i+1} D^{n-i-1} y_{f}\right]-\bar{\delta}_{0}^{T} \varphi_{0} .
\end{aligned}
$$

Thus, the substitution of the above identities, together with (2.17), yields the following extended auxiliary dynamic system which describes the combination of the closed-loop dynamics and control law:

$$
\begin{aligned}
& \dot{x}=A x+w, \\
& \dot{z}=A z+w_{1},
\end{aligned}
$$


with

$$
\begin{aligned}
& w=\left[e+\varepsilon_{0}^{T} i_{\varphi}-\bar{\delta}_{0}^{T} \varphi_{0}, 0\right]^{T}=\bar{w}+w_{1}, \\
& \bar{w}=\left[-\bar{\delta}_{0}^{T} \varphi_{0}, 0\right]^{T}, \quad w_{1}=\left[e+\varepsilon_{0}^{T} i_{\varphi}, 0\right]^{T}, \\
& A(t)=\left[\begin{array}{ccc} 
& \bar{p}^{T} & \\
I_{n-1} & \vdots & 0 \\
\cdots & \cdots & \cdots \\
& \bar{v}^{T} & \\
0 & \vdots & I_{n-1}
\end{array}\right], \quad \bar{p}= \begin{cases}\bar{p}^{(1)} & \text { if } m \leq n-1, \\
\bar{p}^{(2)} & \text { if } m=n,\end{cases} \\
& \bar{p}^{(1)^{T}}=[-\bar{a}_{1},-\bar{a}_{2}, \ldots,-\bar{a}_{n}, \overbrace{0, \ldots, 0}^{n-m-1}, \bar{b}_{0}, \bar{b}_{1}, \ldots, \bar{b}_{m}], \\
& \bar{p}^{(2)^{T}}=\left[-\left(\bar{a}_{1}+\bar{b}_{0} r_{0}\right),-\left(\bar{a}_{2}+\bar{b}_{0} r_{1}\right), \ldots,-\left(\bar{a}_{n}+\bar{b}_{0} r_{n-1}\right),\left(\bar{b}_{1}-\bar{b}_{0} s_{1}\right)\right. \text {, } \\
& \left.\left(\bar{b}_{2}-\bar{b}_{0} s_{2}\right), \ldots,\left(\bar{b}_{n}-\bar{b}_{0} s_{n}\right)\right] \text {, } \\
& \bar{v}^{T}=\left[r_{0}, r_{1}, \ldots, r_{n}, s_{1}, s_{2}, \ldots, s_{n}\right],
\end{aligned}
$$

with $x(0)=z(0)=x_{0}, \quad x=\left(D^{n-1} y_{f}, \ldots, D y_{f}, y_{f}, D^{n-1} u_{f}, \ldots, D u_{f}, u_{f}\right)^{T}$, and $\varphi_{0}=$ $\left(D^{n-1} y_{f}, \ldots, D y_{f}, y_{f}, D^{n} u_{f}, D^{n-1} u_{f}, \ldots, D u_{f}, u_{f}\right)^{T}$. The proof of boundedness and convergence to zero of the input, output, their filtered versions, and the time-derivatives of those ones up to $(n-1)$ th order of the closed-loop system is immediate by first proving that (A.12b) is asymptotically stable in the large. Thus, by vector construction, $\left|D^{n} u_{f}\right| \leq$ $K^{\prime}\|x\|$ from the controller equation (2.17) and, then, $\left\|\varphi_{0}\right\| \leq \operatorname{Max}\left(\left|D^{n} u_{f}\right|,\|x\|\right) \leq K\|x\|$ with $K=1+K^{\prime}$. Note from (A.12b) and (2.19a) that all the eigenvalues of $A(t)$ are less than or equal to $(-\sigma)$ for some real constant $\sigma>0$ which is less than or equal to the minimum absolute value of the roots of the strictly Hurwitz $C^{*}(D)$-polynomial for all $t \geq 0$ (equality applies when both roots are distinct $[1,7]$ ). Also, $A(t)$ is uniformly bounded and, furthermore, $\int_{t}^{t+T_{0}}\|\dot{A}(\tau)\| d \tau \leq \mu T_{0}+\mu_{0}^{\prime}$ for positive constants $\mu$ and $\mu_{0}$, for all $t \geq 0$, and some finite $T_{0}$. This follows directly in the absence of modification on the integration interval since the time-derivative of the estimates and controller parameters are bounded as follows from Theorem 3.2. Assume that there are $\infty>s_{t} \geq 0$ modification switches on $\left[t, t+T_{0}\right]$. Their number is finite since the integration interval is finite and $\left|\operatorname{Det}\left(S\left(\theta_{0}\right)\right)\right|$ is a continuous function of time so that existing switches are isolated (i.e., there is no accumulation point of modification switches). Also, their associate discontinuities in $A(t)$ are given by bounded steps whose norms are upper-bounded by a positive finite constant $\bar{k}$ from Theorem 3.2(i) since $\bar{\theta} \in L_{\infty}$. As a result,

$$
\int_{t^{-}}^{\left(t+T_{0}\right)^{+}}\|\dot{A}(\tau)\| d \tau \leq \sum_{i=1}^{s_{t}} \bar{k} \int_{t_{i}^{-}(t)}^{t_{i}^{+}(t)} \partial\left(\tau-t_{i}(t)\right) d \tau+\int_{\bigcup_{j=0}^{s_{t} I_{j}}(t)}\|\dot{A}(\tau)\| d \tau \leq \mu T_{0}+\mu_{0}
$$

with $\mu_{0}=\mu_{0}^{\prime}+\bar{s} \bar{k}<\infty, \infty>\bar{s}=\operatorname{Sup}_{t \geq 0}\left(s_{t}\right), s_{t}$ is a nonnegative integer number, and $\partial(\tau)$ is the Dirac delta function at $\tau=0$. The $t_{(\cdot)}(t)$ instants are the $s_{t}$ separated instants within $\left(t, t+T_{0}\right)$ where the modification switches take place, and for $i \neq 0, s_{t}$ are $s_{t+1}$ 
open intervals where the time-derivative of the modified estimates exists. (If $s_{t}=0$, then $\left.I_{0}(t)=\left(t, t+T_{0}\right].\right)$

Thus, the common unforced version of both time-varying systems (A.12) is exponentially stable in the large (see $[4,16])$. Now, a direct calculus with the differential systems (A.12a) and (A.12b) yields that their solutions are related as follows:

$$
x(t)=z(t)+\int_{0}^{t} \Psi(t, \tau) \bar{w}(\tau) d \tau
$$

with $\psi(t, \tau)$ being the fundamental matrix of the unforced version of (A.12a) and (A.12b), that is, $x(t)=z(t)=\psi(t, 0) x_{0}$ for all $t \geq 0$ if $w \equiv w_{1} \equiv 0$. Since such a system is exponentially stable in the large, one has for any matrix norm that $\|\Psi(t, \tau)\| \leq K_{\Psi} e^{-\sigma(t-\tau)}$ for any $t$ and $\tau$ fulfilling $t \geq \tau \geq 0$. In particular, one has $\|\Psi(t, \tau)\|_{2} \leq e^{-\sigma(t-\tau)}$ (i.e., $K_{\Psi}=$ 1 ) if the spectral matrix norm is used. Since $A(t)$ is exponentially stable and, furthermore, $w_{1} \in L_{\infty} \cap L_{2}$ from (i) and (ii), $z \in L_{\infty} \cap L_{2}, \dot{z} \in L_{\infty} \cap L_{2}$, and $z$ converges exponentially to zero for any bounded initial condition (see [7]). Thus, by taking spectral vector and matrix norms in (A.15), one gets directly from the definition of $\bar{w}$ in (A.12a),

$$
\|x(t)\|_{2} \leq\|z(t)\|_{2}+\int_{0}^{t} e^{-\sigma(t-\tau)}\left\|\bar{\delta}_{0}\right\|_{2}\|x(\tau)\|_{2} d \tau .
$$

Now, define

$$
\begin{gathered}
\bar{z}_{t_{j}, e}=\operatorname{Sup}_{t_{j} \leq t \leq T}\left(\|z(t)\|_{2}\right), \\
\bar{z}=\operatorname{Sup}_{t \in \mathbb{R}_{0}^{+}}\left(\left\|z_{t, e}\right\|_{2}\right)=\operatorname{Sup}_{T \in \mathbb{R}_{0}^{+}}\left(\operatorname{Sup}_{0 \leq t \leq T}\left(\|z(t)\|_{2}\right)\right)
\end{gathered}
$$

for all finite $t_{j} \in \mathbb{Z}_{0}^{+}$and $T \in \mathbb{R}_{0}^{+}$, where $\mathbb{Z}_{0}^{+}$and $\mathbb{R}_{0}^{+}$are the sets of nonnegative integers and real numbers, respectively. Since $z \in L_{\infty} \cap L_{2}$, there exists a sequence of time instants $T_{s}=\left\{t_{k}, k \geq 0\right\}$ with $t_{0}$ sufficiently large (but finite) such that $\bar{z}_{t_{k+1}, e}<\bar{z}_{t_{k}, e} \leq \bar{z}<\infty$ and $\bar{z}_{t_{k}} \rightarrow 0$ as $k \rightarrow \infty$ since $T_{s}$ is a monotonically increasing sequence and $z(t)$ converges to zero asymptotically since it is in $L_{\infty} \cap L_{2}$. Now, if the basic modification scheme (2.9)(2.10) is used, it follows that

$$
\left\|x\left(t_{k}+\tau\right)\right\|_{2} \leq \bar{z}_{t_{k}, e} e^{\left(-\sigma+\bar{\delta}_{0}^{\prime}\right) \tau}<\infty \quad \forall t_{k} \in T_{s}, 0 \leq \tau \leq t_{k+1}-t_{k}
$$

where $\left\|x\left(t_{k}+\tau\right)\right\|_{2} \leq \bar{z}_{t_{k}, e} e^{\left(-\sigma+\bar{\delta}_{0}^{\prime}\right) \tau}<1$, since $\rho^{\prime} \geq 2 \rho$ by applying Bellman-Gronwall lemma to (A.17) (see [1]), provided that $\rho<|\sigma| / 3(n+m)$. Thus, $\left\|x\left(t_{k}+\tau\right)\right\|_{2}<\infty$ and $\left\|\dot{x}\left(t_{k}+\tau\right)\right\|_{2}<\infty$ are uniformly bounded from (A.18), the boundedness of the estimation error, $\bar{\delta}_{0}^{\prime}$, and (A.12)-(A.13). One has, in addition, from (A.18) that $x\left(t_{k}+\tau\right) \rightarrow 0$ for all $\tau \in\left[t_{k}, t_{k+1}\right)$ as $k \rightarrow \infty$ since $\bar{\delta}_{0}^{\prime}$ is bounded from (i). As a result, $x \in L_{\infty}, \dot{x} \in L_{\infty}$, and $x \rightarrow 0, \dot{x} \in L_{\infty}$ as $t \rightarrow \infty$. Thus, the proof of (iv) follows for the basic modification scheme from the calculation of the solution $x:[0, \infty) \rightarrow \mathbb{R}^{2 n}$ to (A.12a) for any initial conditions. 
If the alternative modification scheme of (2.10a)-(2.10c) is used, then a new upperbound for $\bar{\delta}_{0}^{\prime}$ has to be fixed as follows. Direct calculations with (2.12)-(2.13) yield $\left\|\bar{\delta}_{0}\right\|_{2} \leq\left((1-\alpha)^{2}\left(\sum_{i=1}^{n} a_{i}^{2}+\sum_{i=0}^{m} b_{i}^{2}\right)+\delta b_{m}^{2}\right)^{1 / 2}$ if Condition C.1 does not hold (with $\delta b_{m}=\beta b_{m}$ or $\left.\delta b_{m}=\beta^{\prime}\right)$. By taking also into account (2.12)-(2.13), one gets

$$
\begin{aligned}
\left\|\bar{\delta}_{0}\right\|_{2} \leq & \left(\frac{\left(1-\rho_{\alpha}\right)^{2}}{\left(\sum_{i=1}^{n}\left|a_{i}\right|+\sum_{i=0}^{m}\left|b_{i}\right|\right)^{2}}\left(\sum_{i=1}^{n}\left|a_{i}\right|+\sum_{i=0}^{m}\left|b_{i}\right|\right)^{2}+\beta^{2} b_{m}^{2}\right)^{1 / 2} \\
\leq & \left(1-\rho_{\alpha}\right)+\beta\left|b_{m}\right| \\
= & 1-\rho_{\alpha}+(1-\alpha)\left(\sum_{i=1}^{n}\left|a_{i}\right|+\sum_{i=0}^{m}\left|b_{i}\right|\right) \\
& +(1-\alpha) \rho_{\beta}-\left|b_{m}\right| \leq 2\left(1-\rho_{\alpha}\right)+\frac{1-\rho_{\alpha}}{\sum_{i=1}^{n}\left|a_{i}\right|+\sum_{i=0}^{m}\left|b_{i}\right|} \rho_{\beta}
\end{aligned}
$$

if $\delta b_{m}=\beta b_{m}$, and

$$
\begin{aligned}
\left\|\bar{\delta}_{0}\right\|_{2} & \leq\left((1-\alpha)^{2}\left(\sum_{i=1}^{n} a_{i}^{2}+\sum_{i=0}^{m} b_{i}^{2}\right)+\beta^{\prime 2}\right)^{1 / 2} \\
& \leq\left((1-\alpha)^{2}+2\left(\sum_{i=1}^{n}\left|a_{i}\right|+\sum_{i=0}^{m}\left|b_{i}\right|\right)^{2}+2\left(\rho_{b}^{\prime}+\left|b_{m}\right|\right)^{2}\right)^{1 / 2} \\
& \leq\left(\left(1-\rho_{\alpha}\right)^{2}+2\left(\sum_{i=1}^{n}\left|a_{i}\right|+\sum_{i=0}^{m}\left|b_{i}\right|\right)^{2}+2\left(\rho_{b}^{\prime}+\varepsilon_{b}\right)^{2}\right)^{1 / 2} .
\end{aligned}
$$

Also, if Condition C.1 does not hold, then the following inequalities hold:

$$
\begin{aligned}
\frac{(n+m)^{1 / 2}-\varepsilon_{0}}{\varepsilon_{0}} & <\left\|S\left(\theta_{0}\right)\right\|_{1}-1 \leq \sum_{i=1}^{n}\left|a_{i}\right|+\sum_{i=0}^{m}\left|b_{i}\right| \\
& <1+\left\|S\left(\theta_{0}\right)\right\|_{\infty} \leq 1+\frac{(n+m)^{-1 / 2}}{\varepsilon_{0}^{\prime}} .
\end{aligned}
$$

The remaining part of the proof follows from (A.18) after substituting (A.20) into (A.19) to calculate upper-bounds $\bar{\delta}_{i}^{\prime}(i=1,2)$ of $\operatorname{Sup}_{t \geq 0}\left(\left\|\bar{\delta}_{0}\right\|_{2}\right)$.

\section{Acknowledgments}

The author is very grateful to DGES and UPV/EHU for their partial support of this work through Project DPI2003-00164 and Grant 9/UPV/EHU 00I06-15263/2003 for Research Groups. He is also grateful to the reviewers for their useful comments which helped to improve the original version of this paper. 


\section{References}

[1] R. Bellman, Methods of Nonlinear Analysis. Vol. 1, Mathematics in Science and Engineering, vol. 61-I, Academic Press, New York, 1970.

[2] H. F. Chen and X.-R. Cao, Controllability is not necessary for adaptive pole placement control, IEEE Trans. Automat. Control 42 (1997), no. 9, 1222-1229.

[3] M. de la Sen, Multirate hybrid adaptive control, IEEE Trans. Automat. Control 31 (1986), no. 6, 582-586.

[4] , Robust adaptive stabilization of time-invariant first-order hybrid systems with covariance resetting, Internat. J. Non-Linear Mech. 33 (1998), no. 1, 47-57.

[5] - On the robust adaptive stabilization of a class of nominally first-order hybrid systems, IEEE Trans. Automat. Control 44 (1999), no. 3, 597-602.

[6] Ph. de Larminat, On the stabilizability condition in indirect adaptive control, Automatica J. IFAC 20 (1984), no. 6, 793-795.

[7] C. A. Desoer and M. Vidyasagar, Feedback Systems: Input-Output Properties, Academic Press, New York, 1975.

[8] V. Etxebarria and M. de la Sen, Adaptive control based on special compensation methods for time-varying systems subject to bounded disturbances, Internat. J. Control 61 (1995), no. 3, 667-694.

[9] P. Eykhoff, System Identification, Parameter and State Estimation, John Wiley \& Sons, London, 1974.

[10] A. Feuer and A. S. Morse, Adaptive control of single-input, single-output linear systems, IEEE Trans. Automat. Control 23 (1978), no. 4, 557-569.

[11] L. Guo, Self-convergence of weighted least-squares with applications to stochastic adaptive control, IEEE Trans. Automat. Control 41 (1996), no. 1, 79-89.

[12] D. Kincaid and W. Cheney, Numerical Analysis, Mathematics of Scientific Computing, Brooks/Cole Publishing, California, 1991.

[13] T.-H. Lee and K. S. Narendra, Stable discrete adaptive control with unknown high-frequency gain, IEEE Trans. Automat. Control 31 (1986), no. 5, 477-479.

[14] N. Luo and M. De la Sen, State feedback sliding mode control of a class of uncertain time delay systems, Proceedings of IEE-D: Control Theory and Applications 140 (1993), no. 4, 261-279.

[15] N. Luo, M. De la Sen, and J. Rodellar, Robust stabilization of a class of uncertain time delay systems in sliding mode, Internat. J. Robust Nonlinear Control 7 (1997), no. 1, 59-74.

[16] R. Middleton, G. C. Goodwin, D. J. Hill, and D. Q. Mayne, Design issues in adaptive control, IEEE Trans. Automat. Control 33 (1988), no. 1, 50-58.

[17] A. S. Morse, Global stability of parameter-adaptive control systems, IEEE Trans. Automat. Control 25 (1980), no. 3, 433-439.

[18] K. S. Narendra, Y.-H. Lin, and L. S. Valavani, Stable adaptive controller design. Part II: proof of stability, IEEE Trans. Automat. Control 25 (1980), no. 3, 440-448.

[19] R. D. Nussbaum, A state approach to the problem of adaptive control assignment, Math. Control Signals Syst. 2 (1989), no. 1, 243-246.

[20] J. M. Ortega, Numerical Analysis. A Second Course, Academic Press, New York, 1972.

M. de la Sen: Instituto de Investigación y Desarrollo de Procesos (IIDP), Departamento de Ingeniería de Sistemas y Automática, Facultad de Ciencias, Universidad del Pais Vasco, Campus de Bizkaia, Aptdo 644 de Bilbao, 48080 Bilbao, Spain

E-mail address: wepdepam@lg.ehu.es 


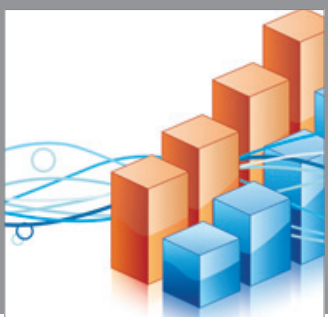

Advances in

Operations Research

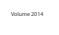

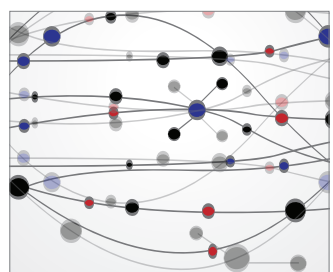

\section{The Scientific} World Journal
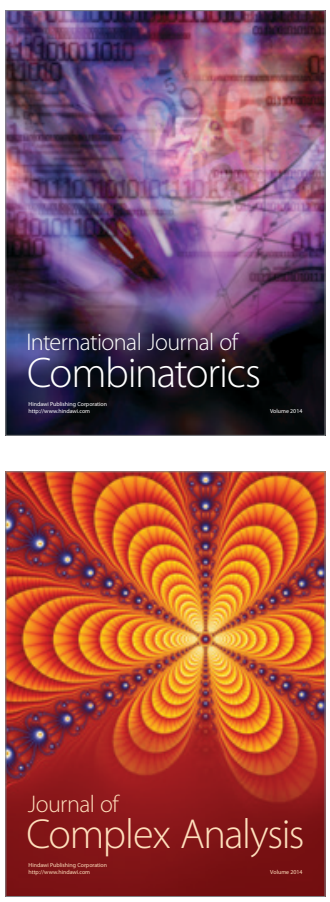

International Journal of

Mathematics and

Mathematical

Sciences
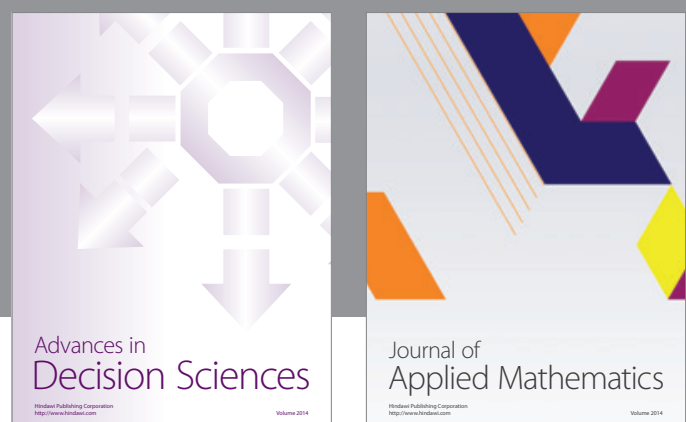

Journal of

Applied Mathematics
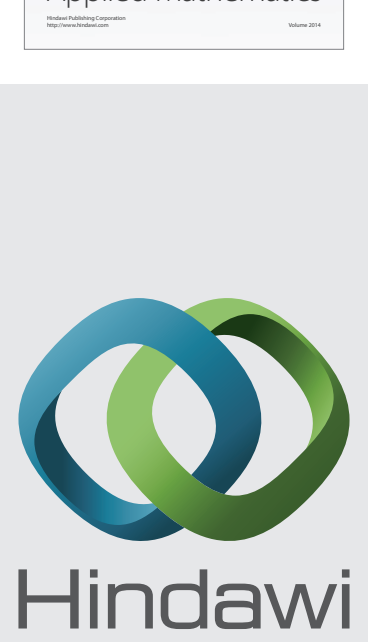

Submit your manuscripts at http://www.hindawi.com
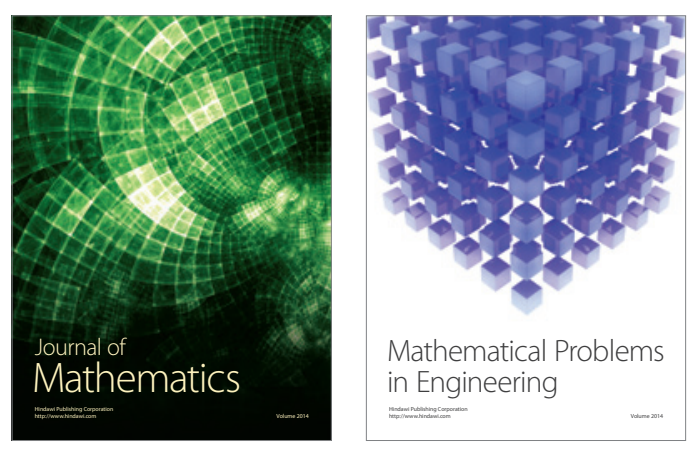

Mathematical Problems in Engineering
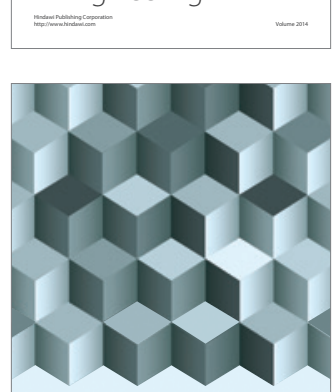

Journal of

Function Spaces
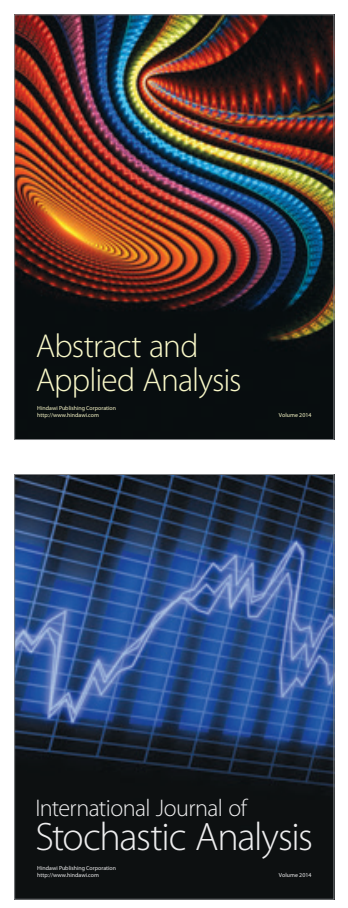

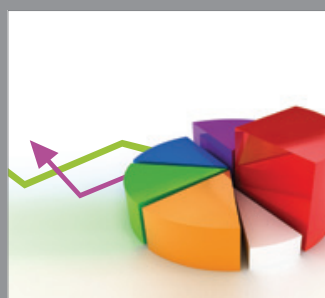

ournal of

Probability and Statistics

Promensencen
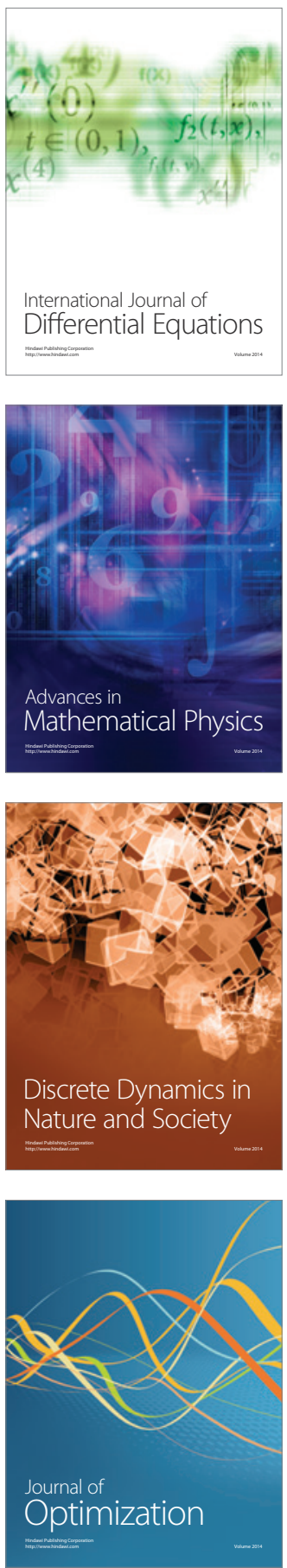